\title{
PREDICTING CHANGES TO SOURCE CODE
}

\author{
A Thesis \\ presented to \\ the Faculty of California Polytechnic State University, \\ San Luis Obispo \\ In Partial Fulfillment \\ of the Requirements for the Degree \\ Master of Science in Computer Science
}

by

Justin Roll

April 2016 
(c) 2016

Justin Roll

ALL RIGHTS RESERVED 


\section{COMMITTEE MEMBERSHIP}

TITLE:

AUTHOR:

DATE SUBMITTED:

April 2016
Predicting Changes to Source Code

Justin Roll

COMMITTEE CHAIR: Davide Falessi, Ph.D.

Associate Professor of Computer Science

COMMITTEE MEMBER: Foaad Khosmood, Ph.D.

Assistant Professor of Computer Science

COMMITTEE MEMBER: Alexander Dekhytar, Ph.D.

Professor of Computer Science 


\title{
ABSTRACT \\ Predicting Changes to Source Code
}

\author{
Justin Roll
}

Organizations typically use issue tracking systems (ITS) such as Jira to plan software releases and assign requirements to developers. Organizations typically also use source control management (SCM) repositories such as Git to track historical changes to a code-base. These ITS and SCM repositories contain valuable data that remains largely untapped. As developers churn through an organization, it becomes expensive for developers to spend time determining which software artifact must be modified to implement a requirement. In this work we created, developed, tested and evaluated a tool called Class Change Predictor, otherwise known as CCP, for predicting which class will implement a requirement. Understanding which class will implement a requirement supports several software engineering tasks such as refactoring and assigning requirements to developers.

CCP is a data-mining tool operating on top of ITS and SCM repositories which gathers a unique combination of metrics. $\mathrm{CCP}$ leverages requirement text to compare current requirements to past requirements and requirements to source code files. CCP performs static analysis on the code-base of each major release of the software artifact. We evaluated CCP on different open source datasets (and the Digital Democracy dataset) by using several machine learning classifiers and pre-processing procedures. Our results show that we can achieve high precision on three out of four datasets. We conclude that accurate class change prediction is feasible, and we propose numerous solutions to increase future accuracy. 


\section{ACKNOWLEDGMENTS}

Thanks to:

- Dr. Davide Falessi, for so much direction and patience.

- Jin Guo from DePaul University, for coding all of the similarity metrics and providing me numerous papers on their meaning.

- Dr. Jane Cleland-Huang from DePaul University, for wisdom and guidance.

- Dr. Alexander Dekhtyar, for letting a humble former humanities major like myself into this program.

- Dr. Foaad Khosmood, for getting me interested in machine learning.

- Nicole L. Smith, for love and support.

- Mom and Dad, just because. 


\section{TABLE OF CONTENTS}

LIST OF TABLES . . . . . . . . . . . . . . . . . . . . . . . . . . . . . . . . . . $\ldots$ xii
LIST OF FIGURES
CHAPTER

1 Introduction . . . . . . . . . . . . . . . . . . 1

1.1 An Overview .......................... 1

1.1 .1 Impact . . . . . . . . . . . . . . 2

1.2 Research Questions ..................... 3

1.2.1 RQ1: What Is The Most Accurate Configuration For The CCP System? . . . . . . . . . . . . . . 3

1.2.1.1 Conjecture .................. 3

1.2.1.2 Hypothesis ................... 3

1.2.2 RQ2: What Is The Overall Accuracy Of Our Classifier On Each Dataset?.................... 3

1.2.2.1 Conjecture ................... 4

1.2.2.2 Hypothesis . . . . . . . . . . . . . 4

1.2.3 RQ3: How Does The CCP Classifier Compare To Other Similar Studies? . . . . . . . . . . . . . . 4

1.2.3.1 Conjecture ................... 4

1.2.3.2 Hypothesis . . . . . . . . . . . . . . . . 4

2 Background and Related Work . . . . . . . . . . . . . 5

2.1 Background ........................... 5

2.1.1 Static Analysis . . . . . . . . . . . . 5

2.1.2 Object-oriented Complexity ............ 5

2.1.3 Natural Language Processing Techniques . . . . . . . . . . . 6

2.1.3.1 Pre-processing ................ 6

2.1.3.2 Vector Space Model . . . . . . . . . . . . 6

2.1.3.3 Document Similarity . . . . . . . . . . . . 7

2.2 Classification . . . . . . . . . . . . . . . . 9

2.2.1 Supervised Machine Learning . . . . . . . . . . . . . . 10 
2.3 Related Work . . . . . . . . . . . . . . . . . . . . . . . 10

2.3.1 Source Code Traceability . . . . . . . . . . . . . . . . 10

2.3.1.1 Event-based Traceability . . . . . . . . . . . . 10

2.3.1.2 Traceability With Machine Learning Classifiers . . . 11

2.3.1.3 Relation To Our Project . . . . . . . . . . . . . . . 12

$2.3 .2 \quad$ Impact Analysis . . . . . . . . . . . . . . . . . . . . . . 12

2.3 .3 Manual Impact Analysis . . . . . . . . . . . . . . . . . 13

2.3.4 Automated Impact Analysis . . . . . . . . . . . . . . . . 14

2.3.4.1 Predicting Class Changes Using Co-change History . 15

2.3.4.2 Predicting Software Changes From Requirements . . 16

2.3.5 Relation To Our Project . . . . . . . . . . . . . . . . 17

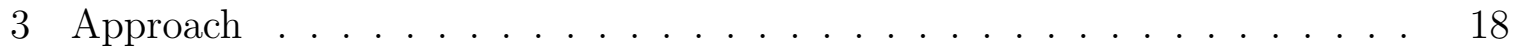

3.1 Linking Process $\ldots \ldots \ldots \ldots \ldots$

3.1.1 From RequirementId To Commit . . . . . . . . . . . . . 19

3.1.2 Determining Touched And Untouched Classes For Each Requirement . . . . . . . . . . . . . . . . . . 21

3.1 .3 Determining Releases . . . . . . . . . . . . . . . . . . . . . . . 22

3.2 Natural Language Processing . . . . . . . . . . . . . . . 23

3.2 .1 Pre-processing . . . . . . . . . . . . . . . . . . . . 23

3.2 .2 Requirement Similarity . . . . . . . . . . . . . . . . . . . . . . 24

3.3 Temporal Locality Calculation . . . . . . . . . . . . . . . . . 28

3.3 .1 Simple Modification $\%$. . . . . . . . . . . . . . . 28

3.3.2 Linear Temporal Locality Modification Score . . . . . . . . . 29

3.3.3 Logarithmic Temporal Locality Modification Score . . . . . . . 29

3.3 .4 Example Calculations . . . . . . . . . . . . . . . . . . . 30

3.4 ML Classifier . . . . . . . . . . . . . . . . . . . . . . . . 32

3.4.1 Feature Pre-processing Configurations _. . . . . . . . . 33

3.4.1.1 Principal Component Analysis . . . . . . . . . 33

3.4.1.2 Top 5 Information Gain . . . . . . . . . . . . . 34

3.4 .2 Classifiers . . . . . . . . . . . . . . . . . . . 34

3.4.2.1 Naive Bayes . . . . . . . . . . . . . . . . . . 34

3.4.2.2 Bootstrap Aggregation . . . . . . . . . . . . . 35 
3.4.2.3 Decision Tree . . . . . . . . . . . . . . . 36

3.4.2.4 The J48 Decision Tree . . . . . . . . . . . . . 36

3.4.2.5 Random Forest . . . . . . . . . . . . . . . . . 37

3.4 .3 Cross-Validation . . . . . . . . . . . . . . . . 37

4 Class Change Predictor . . . . . . . . . . . . . . . . . . . . . . . 38

4.1 Data Stores . . . . . . . . . . . . . . . . . . . . 38

$4.1 .1 \quad$ Jira $\ldots \ldots \ldots \ldots \ldots \ldots \ldots$

4.1.1.1 Jira Extractor . . . . . . . . . . . . . . . . 40

4.1.1.2 Parsing Jira Data Into Requirements . . . . . . . . 40

4.1 .2 Git . . . . . . . . . . . . . . . . . . . . 44 41

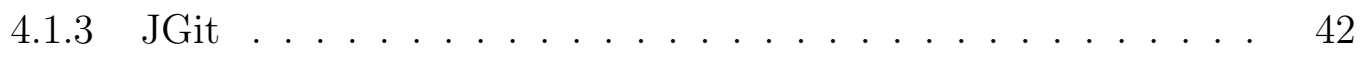

4.1.3.1 Git Extractor . . . . . . . . . . . . . . 42

4.2 Build Tools . . . . . . . . . . . . . . . . . . . . . . . . . . 42

4.2 .1 Maven . . . . . . . . . . . . . . . . . . . . . . 43

4.3 Release Metrics Storage And Tools . . . . . . . . . . . . . . . 43

4.3 .1 SonarQube . . . . . . . . . . . . . . . . . 45

4.3.1.1 SonarQube Executor . . . . . . . . . . . . . . 46

4.3.1.2 SonarQube Scraper . . . . . . . . . . . . . . . . 46

4.3 .2 CKJM Extractor . . . . . . . . . . . . . . . . 47

4.4 Other Technologies . . . . . . . . . . . . . . . . . . . . . 47

4.4 .1 The Semantic Similarity Toolkit . . . . . . . . . . . . . . 47

$4.4 .2 \quad$ Weka . . . . . . . . . . . . . . . . . 48

4.4.2.1 Attribute-Relation File Format . . . . . . . . . . 48

4.5 Configuration And Operation . . . . . . . . . . . . . 48

4.5 .1 Configuration . . . . . . . . . . . . . . . . . . 49

4.5 .2 Commandline Operation . . . . . . . . . . . . . . . 49

4.6 Overall Process . . . . . . . . . . . . . . . . . . . . . . . . . . . 51

4.6 .1 Operation Scenarios _ . . . . . . . . . . . . . . . . . 52

4.6.1.1 Scenario 1: Compute And Serialize Requirements . . 52

4.6.1.2 Scenario 2: Analyze Releases . . . . . . . . . . . 53

4.6.1.3 Scenario 3: Prepare ARFF Files . . . . . . . . . 53

4.6.1.4 Scenario 4: Ensemble. . . . . . . . . . . . . . 53 


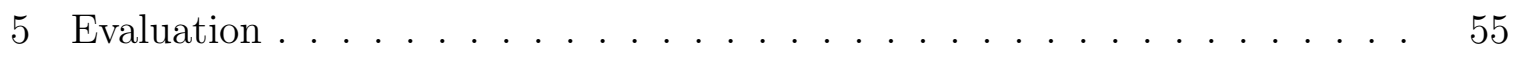

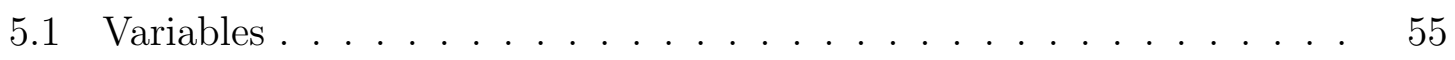

$5.1 .1 \quad$ Dependent . . . . . . . . . . . . . . . . 55

$5.1 .1 .1 \quad$ Precision . . . . . . . . . . . . 55

$5.1 .1 .2 \quad$ Recall . . . . . . . . . . . . . . . 56

$5.1 .1 .3 \quad$ F 0.1 Score . . . . . . . . . . . . . 56

5.1 .2 Independent . . . . . . . . . . . . . . 57

5.1.2.1 SonarQube Violations . . . . . . . . . . . . 57

5.1.2.2 SonarQube Code Complexity . . . . . . . . . . 57

5.1 .2 .3 Lines Of Code . . . . . . . . . . . . . . 58

5.1 .2 .4 Change Frequency . . . . . . . . . . . 58

5.1.2.5 Linear Temporal Locality . . . . . . . . . . . 58

5.1 .3 Logarithmic Temporal Locality . . . . . . . . . . . . . 58

5.1.3.1 CKJM WMC: Weighted Methods Per Class . . . . . 59

5.1.3.2 CKJM DIT: Depth Of Inheritance Tree . . . . . . 59

5.1.3.3 CKJM NOC: Number Of Children . . . . . . . . . 59

5.1.3.4 CKJM CBO: Coupling Between Object Classes . . . 59

5.1.3.5 CKJM RFC: Response For A Class . . . . . . . . . 60

5.1.3.6 CKJM LCOM: Lack Of Cohesion In Methods . . . . 60

5.1.3.7 CKJM Ca: Afferent Couplings _. . . . . . . . . 60

5.1.3.8 CKJM NPM: Number Of Public Methods . . . . . . 61

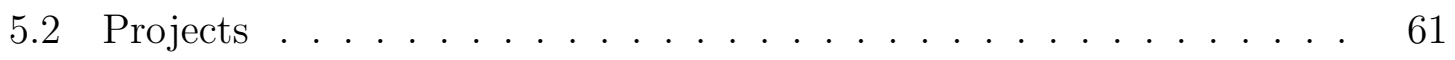

5.2 .1 Selected Projects . . . . . . . . . . . . . . 62

5.2.1.1 Apache Tika . . . . . . . . . . . . . . . . 62

$5.2 .1 .2 \quad$ Apache Isis . . . . . . . . . . . . . . . . . 63

5.2.1.3 Apache Accumulo . . . . . . . . . . . . 63

5.2.1.4 Digital Democracy . . . . . . . . . . . . . . 63

5.3 Results . . . . . . . . . . . . . . . . . . . 63

5.3.1 RQ1: What Is The Best Configuration Of Our Approach? . . 64

5.3.1.1 Best Configuration . . . . . . . . . . . . 65

5.3.1.2 Best Pre-processing Configuration . . . . . . . . 65

5.3.1.3 Best Classifier Configuration _. . . . . . . . . 67 
5.4 RQ2: What Was The Overall Accuracy Of Our Approach? . . . . . . 67

5.5 RQ3: How Does Our Model Compare To Others? . . . . . . . . . . . 69

5.5.1 Comparison To Random Approach . . . . . . . . . . . . . . . 69

5.5.2 Comparison To Previous Studies . . . . . . . . . . . 70

5.5.3 Analysis ...................... . . 70

6 Conclusion And Future Work _. . . . . . . . . . . . . . . . . . 73

6.1 Threats To Validity . . . . . . . . . . . . . . . 74

6.2 Future Work . . . . . . . . . . . . . . . . . 74

6.2.1 Deeper Analysis Of The Results . . . . . . . . . . . . . . 75

6.2.1.1 Compare The Accuracy Of Estimates Made By Using A Single Set Of Metrics Versus The Complete Set . . 75

6.2.1.2 Compare The Accuracy Of Estimates Made By Using Single Metrics Versus The Complete Set Of Metrics . 75

6.2.1.3 Compare The Accuracy Of Our Approach When Using Only Data Related To The First Requirement Versus The Entire Dataset . . . . . . . . . . . . . . 75

6.2.1.4 Find Better Temporal Locality Scores . . . . . . . 76

6.2.1.5 Analyze The Locality Of Changes In The Different Datasets . . . . . . . . . . . 76

6.2.1.6 Identify Other Dataset Characteristics Impacting The Accuracy Of Our Estimates . . . . . . . . . . 77

6.2 .2 Approach Improvements . . . . . . . . . . . . . . . . . . . . 77

6.2.2.1 Leveraging Architecture Relations Information . . . . 77

6.2.2.2 Leveraging Co-changes Information . . . . . . . . 78

6.2.2.3 Pre-process The Requirements For Their Quality . . 78

6.2.2.4 Leveraging Interconnected Frequency Information: Highest Frequency Of A Connected Class . . . . . . . . 79

6.2.2.5 Leveraging Interconnected Complexity Information . 79

6.2.2.6 Measuring All Metrics For Every Revision Rather Than For Every Release . . . . . . . . . . . . . . . 79

6.2.3 Raised Research Questions: Measuring The Usefulness Of Our Approach In Practice . . . . . . . . . . . . 80

6.2.3.1 Release Planning: What is The Reduction Of Defects Provided By Minimizing The Number Of Developers Touching The Same Class? . . . . . . . . . . 80 
6.2.3.2 Refactoring: How Many Refactoring Decisions Could Be Avoided Or Improved By Using Our Approach? . 81

6.2.3.3 Effort Estimation: What Is The Improved Accuracy Of Effort Estimation Provided By Our Approach? . . 81

6.2.3.4 Defect Estimation: What Is The Improved Accuracy Of Defects Estimation Provided By Our Approach? . 82

6.3 Final Thoughts . . . . . . . . . . . . . . . . . . . . 82

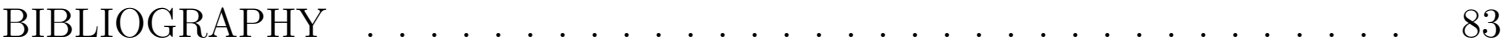
APPENDICES

A Example Class Change . . . . . . . . . . . . . . . . . . . 91

B XML Configuration Example . . . . . . . . . . . . . . 94

C Weka Configuration . . . . . . . . . . . . . . . . . 96

D Features ............................... 98 


\section{LIST OF TABLES}

Table

3.1 Example Of Temporal Locality Result For Three Classes . . . . . . 31

3.2 Temporal Locality Example . . . . . . . . . . . . . . . 33

4.1 Configuration Options ...................... 49

4.2 Command-line Flags . . . . . . . . . . . . . . . . . 50

5.1 Statistics For The 4 Datasets . . . . . . . . . . . . . . 62

5.2 Highest F0.5 Scores By Feature And Classifier . . . . . . . . . 66

5.3 Accuracy Per Dataset .................... 68

C.1 Weka Parameters . . . . . . . . . . . . . . 97

D.1 Requirements To Code Metrics . . . . . . . . . . . . . . . 99

D.2 Textual Similarity Methods To Compute Requirements To Class Associations .................... 100

D.3 Temporal Locality Metrics . . . . . . . . . . . . . . . . . . . . 101

D.4 Source Code Coupling, Cohesion, And Complexity Metrics . . . . . 102 


\section{LIST OF FIGURES}

Figure

3.1 Relationship Between Requirements And Commits . . . . . . . . 19

3.2 Requirement And Commit Life Cycle . . . . . . . . . . . . . . 22

3.3 Dataset Visual . . . . . . . . . . . . . . . . . . 23

3.4 Requirement To Requirement Similarity Example . . . . . . . . . . 26

$4.1 \quad$ System Workflow . . . . . . . . . . . . . . . . . . . . . . . 39

4.2 A Maven Dependency Graph . . . . . . . . . . . . . . . . 44

4.3 How The System Snapshots Releases . . . . . . . . . . . . . . . . . 45

4.4 Map Of Metric Storage Structure . . . . . . . . . . . . . . 45

5.1 Configuration Comparison . . . . . . . . . . . . 65

5.2 Configuration Table . . . . . . . . . . . . . . 66

5.3 Evaluation Data Across All 4 Datasets . . . . . . . . . . . . 69

5.4 Comparison To Random . . . . . . . . . . . . . . . 71

5.5 Comparison To Previous Studies . . . . . . . . . . . . . . 72

A.1 First Modification . . . . . . . . . . . . . . . 91

A.2 Large Amount Of Modifications . . . . . . . . . . . . . . . . . 92

A.3 Many IO-related Modifications . . . . . . . . . . . . . . . 92

A.4 Final Modification .................. 93 


\section{Chapter 1}

\section{INTRODUCTION}

\subsection{An Overview}

In the past quarter century, software has grown from a minor niche to an industry worth hundreds of billions of dollars [65]. Complex software systems are relied upon for every facet of business, and, for many people, software use is intertwined with every-day life. People use software when they contact their friends on social media, when finding directions to a near-by restaurant, and even when starting their car. The high demand for software is exacerbated by the difficulty required in developing complex software systems.

In addition to technical knowledge, software engineering, like other engineering disciplines, typically requires planning. In recent years, agile programming has emerged as a popular software engineering methodology [35]. Under agile methodologies, software is developed piece by piece, using some type of issue-tracking system such as Atlassian's Jira to track development tasks[10]. Software Development Managers, and sometimes the software developers themselves, write requirements which

must be implemented by the software, then post them on an issue tracking system. Once a developer finishes making a code change, she commits it to a source control management system for safe-keeping. Later in the project life-cycle, a thoroughly completed series of software changes is then packaged into a release for public consumption.

Even programmers possessing the technical knowledge required to work on a new software project must often take time to obtain the proper domain knowledge of a software system. A newly hired programmer often has to spend a great deal of time 
pouring over design documentation, F.A.Q.s, and project wikis. If a programmer is asked to make a certain change to a piece of software, the programmer often has to dive into the organization's project management software, finding previous requirements and checking the code changes required to implement them.

Developers also have to make numerous decisions related to the refactoring of software. Refactoring refers to the process of making non-functional changes to software [55]. Refactoring often involves changes such as reducing the complexity of code, making the code more readable, and making code more modular. Refactoring activities can often reduce the accumulated technical debt of a project, at the cost of some lost immediate work [55].

\subsubsection{Impact}

Due to the trade-off of immediate vs. long-term costs, developers are often at a loss as to when to perform a specific refactoring activity. If there is a high likelyhood that a code fragment will change in the future, it can be assumed that there will be benefits to refactoring, as presumably refactoring of the code would reduce the effort required for future changes. However, if the code fragment is unlikely to be modified in the future, it is less useful to refactor it in the present [55].

Developers who can accurately predict what software artifacts will be modified as a result of a requirement are termed domain experts, and often spend valuable development time training new hires. But what if this process could be automated? If a system could be built that would immediately make a code change prediction based on the text of a requirement and the state of the current code-base, this would save organizations valuable time and money.

In response to this need, we introduce Class Change Predictor, or CCP. CCP leverages textual requirement data to compare requirements to other requirements 
and requirements to code. CCP records the state of the code-base as of each major release of the software artifact. CCP then trains a machine learning classifier using textual similarity measures, static analysis metrics, object-oriented complexity metrics, and code change history.

\section{$1.2 \quad$ Research Questions}

In this study, we answer three main research questions. Listed below are the research questions, our conjecture behind them, and our hypotheses.

\subsubsection{RQ1: What Is The Most Accurate Configuration For The CCP System?}

We attempt to maximize the accuracy of CCP by testing with multiple machine learning classifiers and feature sets.

\subsubsection{Conjecture}

Data models often need tuning in order to reach peak accuracy. By testing CCP with several different configurations, we are more likely to find the optimal model.

\subsubsection{Hypothesis}

CCP's accuracy is significantly higher with an optimal feature configuration.

\subsubsection{RQ2: What Is The Overall Accuracy Of Our Classifier On Each Dataset?}

Thorough testing is paramount in generating a high-quality machine learning classifier. Machine learning classifiers should be tested with metrics appropriate to domain needs. 


\subsubsection{Conjecture}

If the CCP tool falsely tells a developer that a class needs to be changed, the developer wastes valuable time before realizing that the class does not need modification. When building CCP, we prioritize accuracy measures that penalize false positives and reward true positives.

\subsubsection{Hypothesis}

CCP is trained with features that are informative. CCP's accuracy measures are high on a variety of datasets.

\subsubsection{RQ3: How Does The CCP Classifier Compare To Other Similar Studies?}

We compare our classifier with multiple related systems in order to properly assess performance.

\subsubsection{Conjecture}

In order for CCP to be useful in industry, it must provide results that are intelligent. CCP must perform significantly better than a random classifier. To further gauge the results, we compare CCP with previous studies on class change prediction.

\subsubsection{Hypothesis}

CCP's accuracy metrics are significantly better than a random classifier overall. CCP provides results that are competitive with prior studies. 
Chapter 2

\section{BACKGROUND AND RELATED WORK}

\subsection{Background}

This section provides technical background on the various concepts related to the creation of CCP. It also introduces concepts that are critical to understanding outside research related to the $\mathrm{CCP}$ project.

\subsubsection{Static Analysis}

Static analysis in terms of software engineering refers to the process of analyzing code without actually running it. There is significant evidence that static analysis is effective in finding faults in software [73]. Developers often run static analysis utilities such as FindBugs at every build iteration in order to find issues such as unused variables, endless loops, and casting errors [7]. Organizations run static analysis tools such as SonarQube at a larger scale, monitoring their entire code-base for quality [67].

\subsubsection{Object-oriented Complexity}

Software artifacts in object-oriented systems can have numerous dependencies. Classes inherit data from their parent classes and are linked to by other classes. A class could have ten lines of code, but many more dependencies. Thus, researchers Kidamberer and Kemberer devised several object-oriented metrics designed to illustrate the complexity and coupling of classes [27]. Classes with high scores in these metrics often have ripple effects - modifying one of these classes can mean modifying several dependent classes. 


\subsubsection{Natural Language Processing Techniques}

Natural Language Processing is the study of the synthesis between computer and human language [19]. NLP is a broad field, incorporating linguistics, statistics, and computer science. NLP is applied to such problems as automated translation, textual similarity, and artificial intelligence.

\subsubsection{Pre-processing}

Researchers generally use pre-processing techniques to format and filter text. They often start by removing "stop words" - common articles in the English language such as "on", "the", and "a" [52]. These common words often contribute noisy data that

provides little information. Researchers often stem words down to their latin roots; "biology" becomes "bio", "aquamarine" becomes "aqua". The logic behind this is that words such as "agreement" and "agreeing" should have the same base meaning $[52]$.

\subsubsection{Vector Space Model}

A Vector Space Model refers to the organization of documents into a matrix of equal length vectors [18]. Each vector of the matrix represents a count of index terms. If there are one thousand unique words or index terms across all documents, then each vector will have one thousand elements [18]. Once these vectors have been computed, documents can be compared using a variety of similarity measures. 


\subsubsection{Document Similarity}

When performing software traceability studies, researchers often use document similarity measures such as Jensen-Shannen Divergence [13]. We used several different techniques in calculating requirement to code and code to code similarity measures.

Jensen-Shannen Divergence Based on the Kullback-Leibler divergence measure, the Jensen-Shannen model is commonly used in traceability studies, bio-informatics, and the social sciences [61]. Because it is a symmetric measure, the square root can be taken to compute the Jensen-Shanning distance. Each document is organized into probability distributions using techniques such as TF-IDF. The documents are then ranked by the distance of their probability distributions relative to other documents. The difference measure between two documents is known as the divergence [61].

$$
\begin{gathered}
K L D(x, y)=x i \log x i / y i \\
J S D(x, y)=1 / 2 K L D(x, m)+1 / 2 K L D(y, m)
\end{gathered}
$$

TF-IDF And Cosine Similarity TF-IDF is an algorithm that is frequently used for information retrieval and document classification tasks. TF-IDF stands for term frequency times inverse document frequency. The algorithm works by taking the count of words in each document (term frequency), and then for each of those words, checking how often it appears in all of the documents. If a word appears in every document, that means that the word doesnt provide much information about the uniqueness of the document and is weighted lower by the algorithm [52].

Finally, once a vector has been created for each document, it is often useful to know how similar documents are to each other. Cosine similarity is a popular and

effective tool to perform this comparison. For a document $\mathrm{d} 1$ and a document $\mathrm{d} 2$, the 
angle between the vectors of $\mathrm{d} 1$ and $\mathrm{d} 2$ is calculated as aD1D2. The cosine of this angle is then taken[52].

Semantic Similarity Comparators Another technique that is often used is called Semantic Similarity Comparison. In this method, researchers use databases like WordNet to determine the similarity between every pair of words in two documents[45]. They then use an algorithm to systematically assess the total similarity score between the two documents[45]. The algorithm used is henceforth referred to as a comparator. We made use of the Optimum Comparator, Corley Mihalcea Comparer Comparator, and Bleu Comparator in our system.

Greedy Comparator In the Greedy Comparator, every word in a document one is compared to every word in another document, and a similarity score is assigned. All words in document one are then iterated through, and the highest similarity score between the word in document one and any word in document two is recorded [66]. All of the word scores are then added up and normalized to output a score between 0 and 1.

Optimum Comparator As in other algorithms, the Optimum Comparator starts by comparing every word in document one to every word on another document. The algorithm then attempts to answer the question: how can each word from D1 be paired with a word from D2 in such a way that produces the maximum similarity score? The problem is then reduced to a bi-partite graph, with the weights of each edge being a similarity score between two words, and each word being a node. The optimal similarity score can be found by discerning the traversal of the graph with the most total weight. The Kuhn-Munkres method and other algorithms can be used to find this optimal path in polynomial time [66]. 
Corley Mihalcea Comparator Introduced in 2005, the Corley Mihalcea Comparator attempts to combine many word-to-word comparisons in order to produce a single text-to-text comparison between two documents [58]. Like TF-IDF, it weights the specificity of words using their inverse document frequency, so that an article like "be" does not have the same weight as "sheepdog". Every word in document 1 is compared with every word with document 2 , with nouns and verbs being compared via WordNet similarity, and all other parts of speech being compared lexicly. Afterwards, for each word in document one, the optimal similarity score in document 2 is chosen. All of these scores are then added up and flattened into one score. This process is then repeated for document 2 with respect to document 1 , producing another flat score [31]. The two scores are then averaged, producing a final Corley Mihalcea score between 0 and 1 .

Bleu Comparator The Bleu Comparator, invented to evaluate the aptitude of machine translations, works by evaluating the n-gram precision between two texts, penalizing the text for being overly or underly verbose [64]. A score representing the quality of the translation from document D1 to document D2 is outputted on a scale from 0 to 1 . It is our hypothesis that a highly similar requirement pair will be "translatable" to one another, and thus have a high Bleu score[64].

\subsection{Classification}

In the realm of statistics and computer science, classification refers to the process of predicting a label for a piece of data given a set of inputs [54]. The set of input data is generally referred to as a set of features. Data Scientists attempt to manipulate a dataset's features to produce the most accurate classifications. More information on 
the specific machine learning techniques used in CCP can be found in the Approach and Evaluation chapters.

\subsubsection{Supervised Machine Learning}

A supervised machine learning classifier relies on a set of past inputs in order to make future predictions [54]. This process is known as training. Oftentimes, researchers will set aside a chunk of data to use for training, and another, usually smaller chunk for testing.

\subsection{Related Work}

The following is a summary of previous research that is related to the CCP project. Each study is highly related to either impact analysis or traceability.

\subsubsection{Source Code Traceability}

The study of source code traceability attempts to make connections between software design materials and the resulting code [44]. Traceability mechanisms between software architecture and code can ease the burden of software maintenance by making the code easier to understand.

\subsubsection{Event-based Traceability}

Buchgeher and Weinreich introduced the LISA sytem to track and enforce traceabil-

ity. LISA required design engineers to use a semi-structured design language[44]. When software engineers made modifications to the software, they were required to specify which active design decision they were working on. Next, software engineers performed design implementation tasks, and these events were logged. The trace- 
ability target was then evaluated. This differs from our task in that we do want to analyze traceability independently of any proprietary design system or language.

Hammad et al. used a method that used various syntactic techniques to evaluate whether a particular code change altered the software design [43]. They found that changes such as adding or removing classes and methods often correlated with design changes, while changes such as modifying data structures, conditional statements, and loops did not. Their system works by first performing a diff of the code changes, converting the diff to XML, and performing a variety of XPath queries to determine what pieces of the code have changed. The tool then attempts to see what relationships have changed. Our tool will differ from this one in that we want to predict code changes based on requirements, while this tool simply analyzes the code that has changed and categorizes it.

\subsubsection{Traceability With Machine Learning Classifiers}

There have been several studies that have utilized machine learning to find software traceability links, many of them performed by researchers at DePaul University [29]. Cleland-Huang et al. performed an experiment to trace code back to its requirements using an ML classifier. Using government regulatory codes, requirements, and the traces, they were able to achieve of .807 for the Encrypt and Decrypt code, although the algorithm was not this accurate across the board.

Mirakhorli et al. utilized two different classifiers for some of their research at Depaul in 2012 [62]. First, they identified several different tactics or categories: heartbeat, scheduling, resource-pooling, authentication, and audit-trail. They took a code dataset and labeled each piece of code with a tactic for training, then built a classifier which could predict the tactic for a piece of code. They also took several descriptions of the tactics from text-books, labeled them, and built another classifier 
trained on this data. They then created a classifier trained on different sub-roles for each tactic, and it was run after all the classes were initially classified.

In 2014, Cleland-Huang et al. performed a study on the present and future of software traceability. They detailed that most software traceability practices are done manually, which requires extensive time and effort[30]. When traceability is implemented, it is often done in a rushed manner at the end of a product life-cycle in order to meet certification requirements. Cleland-Huang et al. note that it should be the long-term goal of the software engineering community to construct an ubiquitous, automated traceability tool. Many of the current traceability tools are imperfect; they are either tailored for a certain project or lacking in accuracy. They posit that a primary research goal should be to "Develop intelligent tracing solutions which are not constrained by the terms in source and target artifacts, but which understand domain-specific concepts, and can reason intelligently about relationships between artifacts" [30].

\subsubsection{Relation To Our Project}

Our project is related to traceability in that we are, in a sense, attempting to perform some traceability analysis to link requirements back to java classes. Our classifier differs from the DePaul studies in that we are seeking to build a classifier that does not need to be trained on a pre-defined set of requirements; we want it to be trained on a project's specific set of requirements.

\subsubsection{Impact Analysis}

Impact analysis is the study of the work generated by a change request, with the change request being something as formal as a requirements document, or something more informal such as a bug-fix ticket. For example, oftentimes in industry today, 
impact analysis is performed manually by software engineers on a case-by-case basis [26]. The work generated by a change request is referred to as an impact set. An impact set could consist of fine-graned elements as methods and single lines of code, or course-grained elements such as a class file or package. When a change request is made, the initial set of elements thought to need modification is called the starting impact set. Once impact analysis is completed and work has been performed, the set of elements that actually changed is called the actual impact set. This terminology emphasizes the point that there are almost always unintended consequences to a change request - the candidate impact set rarely matches the actual impact set.

\subsubsection{Manual Impact Analysis}

From 1996 to the mid 2000s, Shawn A. Bohner conducted a number of studies on impact analysis. In 1996, Bohner performed a study detailing ways to integrate impact analysis into the development process at the human level [22]. In 2003, Bohner attempted a study of impact analysis in regards to changes to commercial off-theshelp components [21]. Keeping in mind the concepts of the starting impact set (the changes estimated to be made) and the actual impact set (the actual changes), Bohner detailed that software objects could be modeled as a dependency matrix or graph, from which one could look at both indirect and direct impacts. Each Software Lifecycle Object (SLO) could be thought of as being a certain distance away from another SLO. SLOs that are a large distance from each other could be objects in different modules, so making a change to one unlikely to affect the other. SLOs that have a short distance are usually highly dependent on each other. With this measure of distance in mind, Bohner then observed that using 3D visualization techniques was useful in analyzing the actual impact sets. 
In 1998, Mikael Lindvall and Kristian Sandahl performed a study on requirementsdriven impact analysis inspired by Bohner's previous work [57]. They worked with developers of the PMR project over two releases and four years, recording relevant data for impact analysis. Based on the given requirements for a release, the developers predicted the $\mathrm{C}++$ classes in the code-base that were likely to change. The researchers measured the correctness of the predictions in terms of the successful class

predictions (true positives) against unsuccessful predictions (false positives), as well as the completeness of the class predictions, which refers to the number of classes expected to change vs. the number of classes that actually changed. For the first release, the correctness of the predictions was $81 \%$, and the completeness was $31 \%$. For the second release, the correctness was $86.1 \%$ and the completeness was $38.8 \%$. This shows that, in addition to requiring extra time and effort on the part of developers, manual impact analysis does not necessarily yield perfect results; even domain experts of a software often produce candidate sets that are much different from the actual impact sets for a given change.

\subsubsection{Automated Impact Analysis}

In 2005, Tsantalis et al. developed a tool to predict the probability of change for a class using a system's code base and its change history. Given a class's dependencies, summarized by its CKJM metric scores, and its history of change, their system outputted a probability using logistical regression [69].

In 2011 study, Lehnert et al. categorizes the state of the art on impact analysis research [56]. In addition to program slicing and information retrieval techniques, researchers have used Call Graphs, where graphs are composed of function calls extracted from code, execution traces, where the software is analyzed for impact analysis as it runs, Program Dependency Graphs, where dependencies between certain code 
fragments are extracted, Probabilistic Models, using Markov Chains or a Bayesian system to check how likely a change is to modify other code fragments, and History Mining, where entities that often change together are analyzed.

More recently, in 2015, Acharya et al. performed a study on static program slicing and its usefulness in impact analysis [15]. Static program slicing is the process of isolating or slicing the code so that only a certain piece related to a certain function is extracted. Acharya et.al created a tool called IMP which attempted to address common issues with static program slicing tools, such as accuracy and performance. This tool is heavily tied to the CodeSurfer API as well as Visual Studio, so is not ideal for future open source development.

\subsubsection{Predicting Class Changes Using Co-change History}

In 2004, Ying et al. used association rule data-mining techniques along with past class-change history to predict to predict future class changes [72]. Using a Frequency Pattern Tree (FPTree) and a low min-support threshold, they developed an association rule algorithm and ran it on the Eclipse and Mozilla projects. The developer would need to specify one file involved in the changes, and the association rules would recommend the rest. Their tool was essentially a probabilistic recommendation system.

More recently, in 2011 Eski et al. conducted an empirical study on object-oriented metrics in order to predict change-prone classes [38]. They assigned different weights to different code modifications, with something like a method change having a higher weight than a field being added to a class. They computed the weights for each class historically, and the classes with the top $10 \%$ most weighted scores were grouped together as the most changed classes. 


\subsubsection{Predicting Software Changes From Requirements}

There have been a few previous studies which have attempted to predict changes to software based on either requirements, the previous code-base, or a combination of both.

In 2005, Canfora et. al performed experiments attempting to identify the work created, or files changed, as a result of the creation of Bugzilla tickets [26]. To do this, they used information retrieval techniques. They looked at past change requests, and if they were similar to the current change request, they took into account the files those past requests modified. They used NLP techniques such as stemming the words, computing word frequencies, and scoring the documents by relevance, using an input requirement as a search query.

In 2013, Kim et al. worked on a system to predict what source code files would change as a result of bug-fix requests [50]. They used the Mozilla FireFox and Core code repositories as their corpus in tandem with the public Bugzilla database for both. They built what was essentially a two-pass classifier. For the first pass, they hand-tagged bug-fix requests as either "USABLE" or "NOT USABLE", and then trained a classifier based on natural language processing features to predict whether the bug-fix request was usable. On most of the analyzed datasets, at least half of the bug-fixes were filtered out in this manner. They did this because vague bug fix requests will probably yield incorrect information to the end user. For the next pass, they extracted all of the words out of each bug-fix request, stemmed all the words, computed term frequencies, and filtered out all common stop-words. They then used all of this information to create features for classification, along with the metadata of the bug-fix request (system, epic, etc) and then trained the classifier. They claimed $70 \%$ accuracy on data that was actually usable [50]. 


\subsubsection{Relation To Our Project}

Our project is highly related to impact analysis, as on some level, we are trying to predict the work created as a result of a requirement. Because manual impact analysis is such a time-consuming and fault-prone endeavor, we are seeking to perform this impact analysis in an automated fashion. Though our project does not fit neatly into any of the categories named in Lehnert's taxonomy, we do use elements of History Mining (when extracting features related to how likely a class is to change), information retrieval (when gathering various complexity and static metrics for a given class), and probabilistic modeling (given that we train a machine learning classifier) $[56]$.

In particular, our research most closely relates to the studies of Canfora et al. and Kim et al., in that we use past change request history and NLP similarity measures to determine future software artifact changes. Our project also relates to Tsantalis et al., in that we use class dependency and quality analysis to guide our prediction of class changes. 
Chapter 3

APPROACH

Our approach combines NLP, static analysis, CKJM metrics, and temporal locality data into a single machine learning classifier. While there have been numerous studies on traceability and bug prediction, we are not aware of another study that combines both static analysis, object-oriented complexity, and NLP techniques to attempt to predict class changes. We blend elements of both impact analysis and traceability to produce a unique approach.

\subsection{Linking Process}

Unfortunately, most project management tools we tested are not directly linked to an SCM. Thus, the CCP linker must perform some data-processing to determine which data should be connected to which commits.

1. The linker attempts to link each requirement to one or more commits.

2. The linker calculates the approximate set of files present at the time of the requirement, as well as the number of files touched as a result of the requirement.

3. Temporal locality is calculated (with care) for each requirement.

4. CCP then calculates the names and commit IDs of each release.

5. For each release, CCP performs static analysis.

6. For each requirement, its release ID is checked. CCP links release metrics to requirements that are part of that release. 


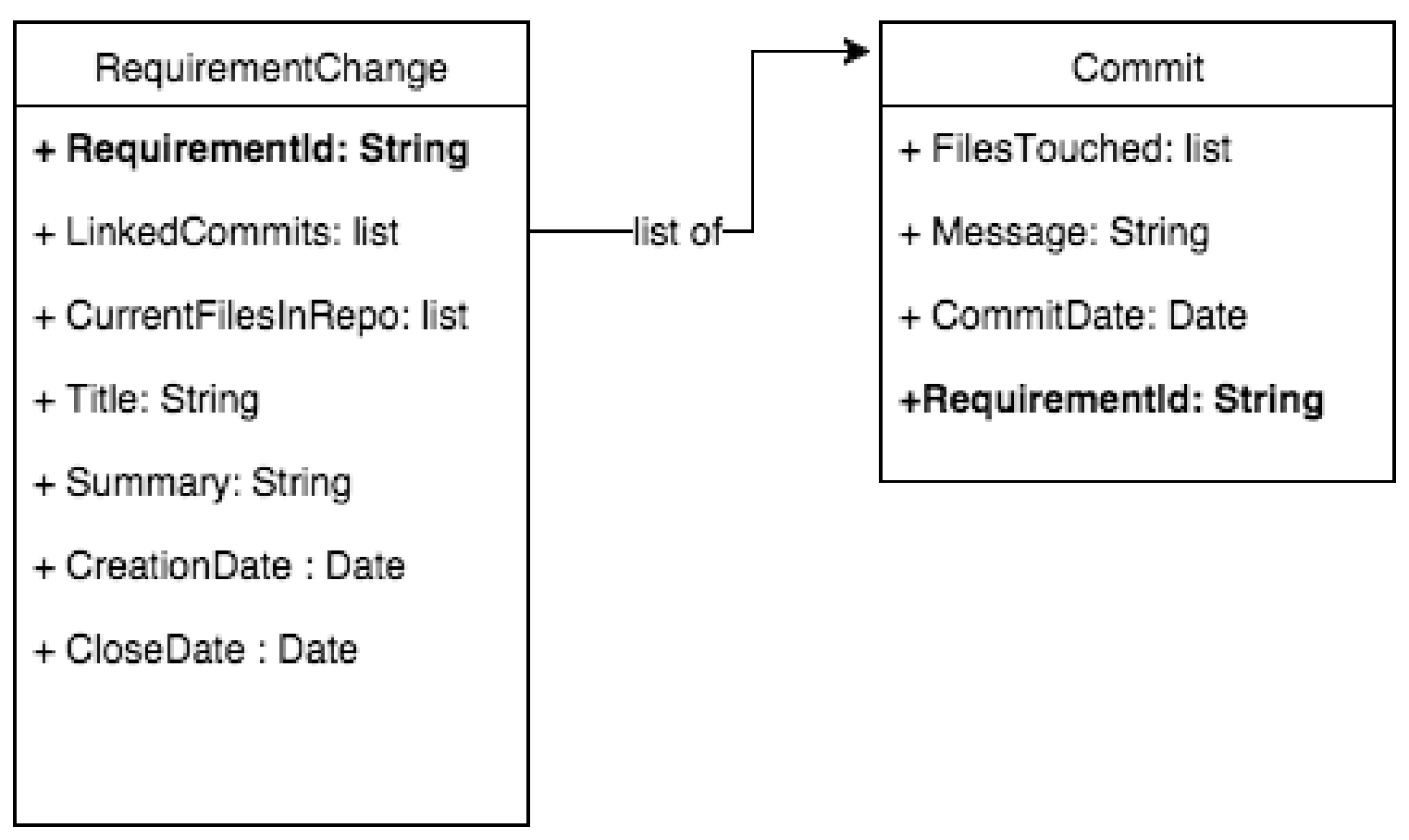

Figure 3.1: Relationship Between Requirements And Commits

7. Any externally computed metrics, such as VSM calculations, are linked in as well.

The most central data structure in the linking process is that of a RequirementChange. A RequirementChange is simply a textual requirement that can be directly linked to source code changes. Once the RequirementChange object construction has been completed, it will contain several linked commits, a list of files that were touched by those commits, and an approximate list of untouched files present in the system at the time of the requirement's creation. Each instance of a class being touched or untouched will be used as a sample in the finished dataset.

\subsubsection{From RequirementId To Commit}

$\mathrm{CCP}$ relies on software developers following specific rules when performing commits: they must specify the full ID of the originating requirement when performing a com- 
mit. So, if a requirement is created named TIKA-11, the commit associated with the requirement must have TIKA-11 in its commit message. Assuming the project is named TIKA, the commit is evaluated with the regular expression .*TIKA-([0$9]+) .^{*}$.

When CCP parses all of the commits, it will check each commit for a valid requirement ID. If a commit does not contain a valid requirement ID, it will not be linked. 
function LinkRequirements $(r, c)$;

Input : A set of requirements and a set of commits $r$ and $c$

Output: RequirementChangeMap

RequirementChangeMap $=$ new

for commit in $c$ do

if commit.containsReqId() then

for requirement in $r$ do

if requirement.id $==$ commit.reqId then

if !RequirementChangeMap.contains(commit.reqId) then

RequirementChangeMap.put(commit.reqId, new RequirementChange

else

end

RequirementChangeMap.get(commit.reqId).link(commit)

else

end

end

else

a

end

end

Algorithm 1: Algorithm For Linking Requirements And Commits

\subsubsection{Determining Touched And Untouched Classes For Each Requirement}

In order to determine the touched files as a result of a requirement, we simply add up the number of changed files in the RequirementChange object's linked commits. We then determine the untouched files present at the time of the requirement by checking out the first commit to implement the requirement, looking at all its files, 


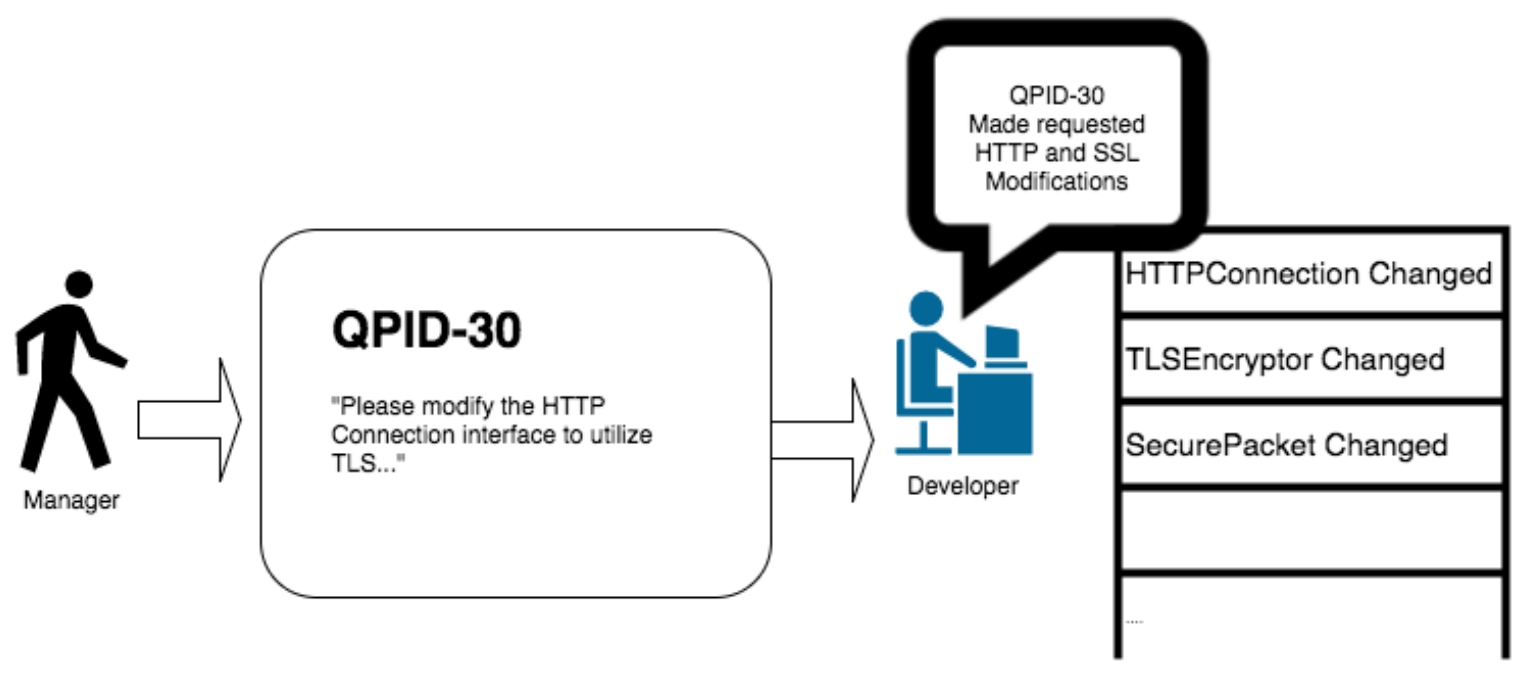

Figure 3.2: Requirement And Commit Life Cycle

then filtering out any files that were created after the requirement date. We filter out any files that were present in our list of touched files.

This is not a perfect procedure, but we found it simpler and less error-prone than other methods.

\subsubsection{Determining Releases}

Determining which commit is the first in a release can be difficult. Sometimes, users do not tag software releases properly in the SCM repository. Or, they create many extraneous "tags" that are not actually releases. We thus employ a heuristic in order to approximate the commit ID of a release. We assume that each requirement in the project management software is marked with a release ID. We then, using our set of linked requirements, look up the commit ID of the first requirement to implement that release and record its parent commit ID. If we did not take the parent commit of the first requirement in a release, we could risk effectively giving requirements data from the future, invalidating our data. 


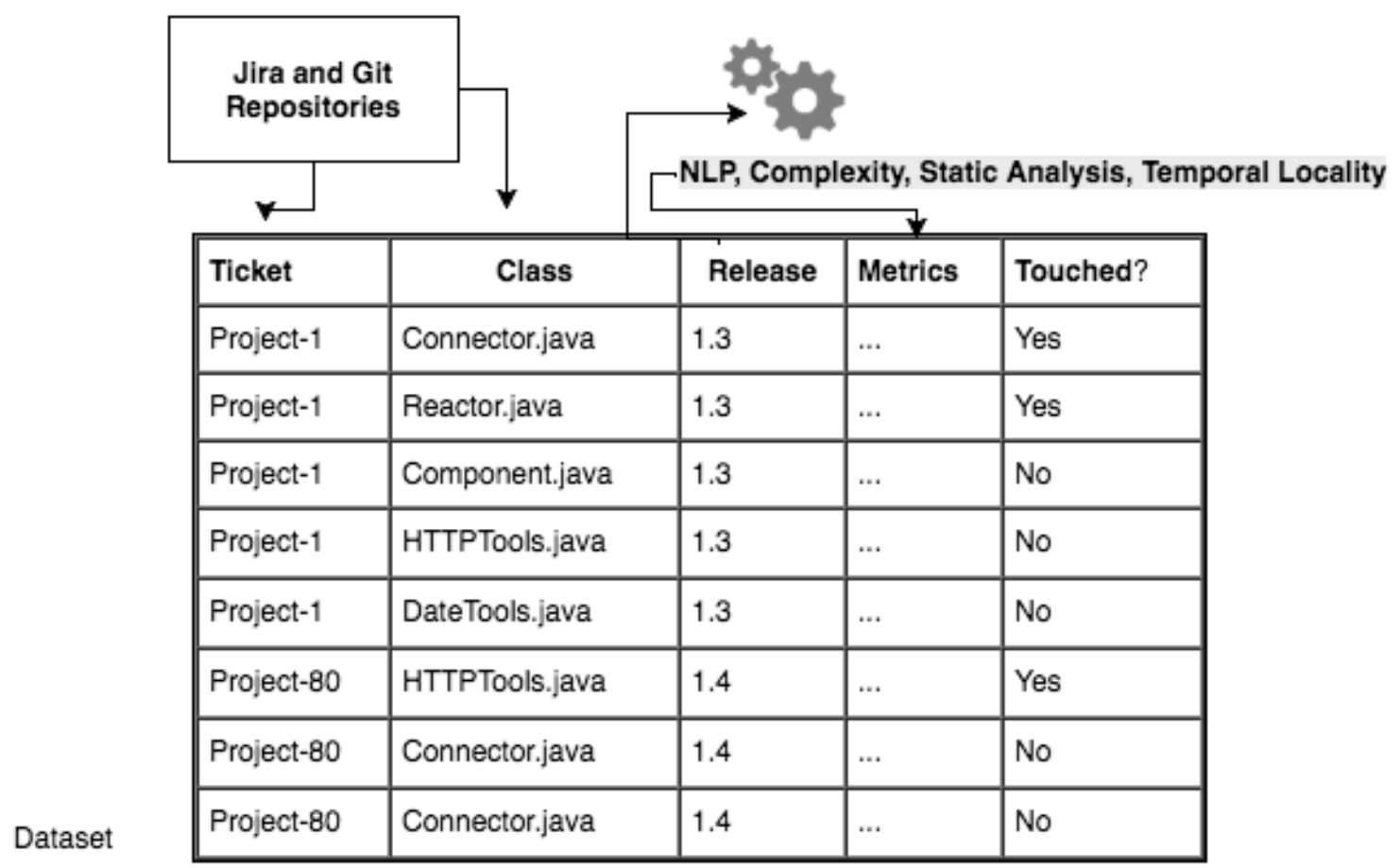

Figure 3.3: Dataset Visual

\subsection{Natural Language Processing}

As the CCP system deals with textual requirement data, we employed sophisticated NLP techniques in order to synthesize this data. Below is a summary of methods used in our textual pre-processing and similarity calculation steps.

\subsubsection{Pre-processing}

Our pre-processing methodology is fairly similar to standard methods[46]. First, we remove any characters not related to the English language, such as '@'. We do this because such characters are often extraneous and don't contain much meaning; they could indicate a requirement as more unique than it actually is.

We then split up 'CamelCase' class, method, and variable names, so "GitExtractor becomes" git extractor, and "bankPostRoutine" becomes "bank post routine" files. 
This is because a requirement will often contain text such as "Please modify the bank post routine to do x". If we simply left "bankPostRoutine" in-tact, the requirement text and the code text wouldn't have the words "bank post routine" in common, and thus we'd miss valuable similarity information.

Finally, we stem each word using the Porter stemmer. The Porter Stemmer works by running a word through a series of rules until the word has been truncated in such a way that no more rules can be applied [46]. For example, consider the word "agreed". The stemmer might have a rule saying that if a word has a vowel, a consonant, and an "eed" ending, remove the "d", which leaves us with "agree". Stemming allows us to reduce different grammatical forms of a word to the word's stem [46]. This allows us to filter words like "agreement" and "agreeing" down to their base stem of "agree". This helps us to accurately match words based on their intended meaning, improving accuracy scores.

\subsubsection{Requirement Similarity}

One of our new research contributions is the ability to historically compare requirements that have modified a class. Every time a class is touched, we make note of the past requirements that the code change implemented. We hypothesized that past requirements that changed a class should be similar to current requirements that modified a class. They will probably at least have certain key-words in common. Below is an example of two requirements which both led to implementation of the same class.

R1:

"We should define what happens in a SortedKeyValueIterator when hasTop, next, getTopKey, and getTopValue are called before init and before seek. We should expect the defined behaviors in tests, and where possible we should enforce those behaviors." 
R2:

"The iterator options for input formats are packed into a single string and delimited with colons and commas. The options aren't escaped, so they cannot contain the delimiter characters. This should be documented and/or fixed."

Keeping a list of the past ten requirements that changed a class (prior to the current requirement), we calculate the semantic similarity comparators of each requirement. We then compute the maximum, median, and average of the top five scores for each requirement. 


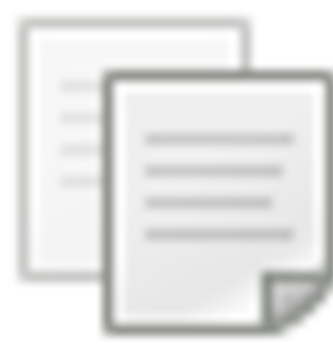

Last 10 Requirements that Touched Class

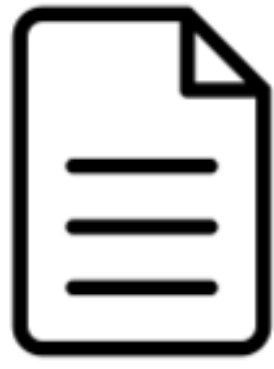

Current Requirement

Produce similarity scores between each of last 10 requirements and current requirements

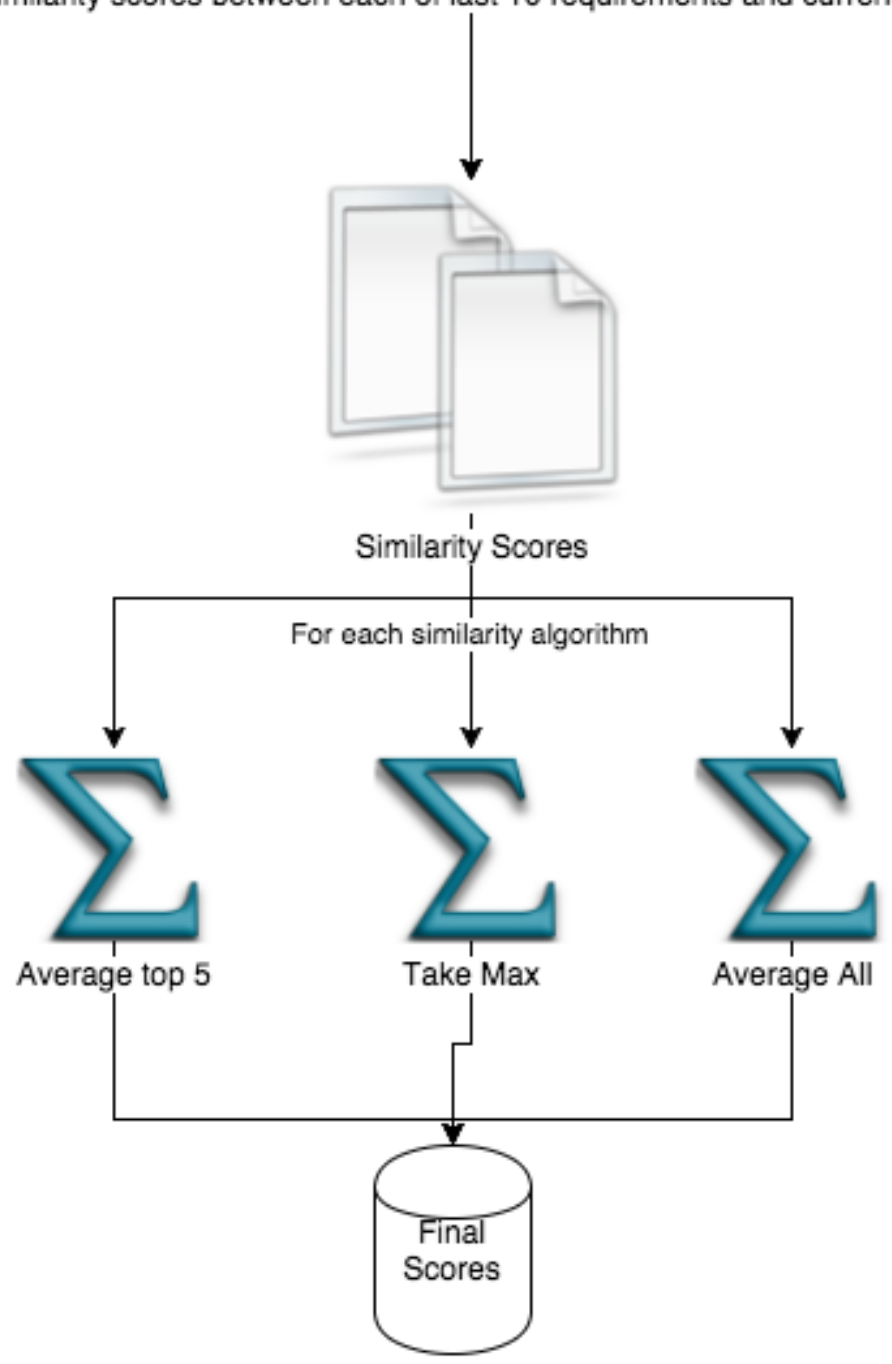

Figure 3.4: Requirement To Requirement Similarity Example 


\section{Compute requirement to requirement similarities}

for all requirement do

for all class in requirement do

last10 $=$ compute last 10 requirements that touched the class

similarities $=$ computeSimilarities (last10)

for all similarityList in similarities do

featureList $=$ new list

featureList.add(average(similarityList, requirement))

featureList.add(averageTopFive(similarityList, requirement))

featureList.add $(\max ($ similarityList, requirement $))$

output(featureList)

end for

end for

end for

computeSimilarities Compute the Similarities using each algorithm

algorithmList $=[$ BlueComparator, CorleyMihalcea, GreedyComparator, OptimumComparator, jensenShannon, VSM]

similarities $=$ new list

for all algorithm in algorithmList do

for all requirement in last10 do

similarities.add(algorithm(requirement, originalRequirement))

end for

end for

return similarities

Algorithm 2: Algorithm For Computing Requirement To Requirement Similarities 


\subsection{Temporal Locality Calculation}

We computed several different features based a class's history of being modified. Our reasoning was that if a class had been modified in the past, it would be more likely to be modified in the future. As you can see from these project results, we found that past modifications do have a correlation with future modifications.

We then wanted to include some concept of temporal locality - which is the concept of more recent modifications being more important than older modifications. Kim et al. pioneered the use of temporal locality in predicting software changes, building a variety of caches to weight the importance of prior software modifications [51]. Bernstein et al. rolled up the number of modifications for a class by month for the most 6 recent months, and used those temporal features to train a defect-predicting classifier[20]. With both of these concepts of temporal locality in mind, we developed two new metrics to measure the temporal locality of recent modifications for each class.

For the below formulas, $\mathrm{k}$ is the number of modifications for the class as well as the index of the oldest modification. The letter $\mathrm{n}$ denotes the total number of commits involving the class, whether it was touched or untouched. The letter i represents number of the current modification, with the most recent commit involving the class having index 1 . The closer the modification is to index 1 , the most recent modification, the more weight it will be given.

\subsubsection{Simple Modification \%}

For the simplest of our history score calculations, we divided the number of times

the class had been touched up until that point in the history by the total number of commits where that class existed. Though this computation was rather simple, we 
found it to be a useful feature.

$$
\frac{k}{n}
$$

\subsubsection{Linear Temporal Locality Modification Score}

For the next of our temporal locality calculations, we incorporated temporal locality. We store a map of each class and its history of modifications, calculating a score that lends more weight to recent class modifications. Given that the most recent commit

index is 1 , the modification of the class at commit index 2 will be given a weight of .5 , the modification at index 3 will be given the weight $1 / 3$, etc. All of these weights will then be divided by the total number of commits where the class had existed up until that point in time.

$$
\frac{\sum_{i=1}^{k} \frac{1}{i}}{n}
$$

\subsubsection{Logarithmic Temporal Locality Modification Score}

We found that on occasion, the previous method gave older modifications too little weight. We found that by instead of dividing by $k$, dividing by $\ln (\mathrm{k})$ when $\mathrm{k}$ is greater or equal to 3 gives us a sufficient amount of temporal locality. The weight decays much more gradually than with the previous method. So, the modification at commit index 4 will be given a weight of $1 / \ln (4)$, the modification at commit index 5 will be given a weight of $1 / \ln (5)$, etc. Again, all weights will then be divided by the total number of commits where the class had existed up until that point in time.

$$
\frac{\sum_{i=3}^{k} \frac{1}{\ln (i)}}{n}
$$




\subsubsection{Example Calculations}

To better understand the calculation of temporal locality scores, it is useful to map out a simple example. Given the table below, six different requirements have been processed, and there are three different classes in the dataset.

For the simple calculation, we see that class A has been touched 4 different times, so the modification percentage would be four divided by six or two thirds. Class $\mathrm{B}$ has been touched three times, and there are a total of six requirements, so the modification score would be one half. For the linear calculation, we start taking into account the temporal localities of the class modifications. Class A has been touched at its sixth oldest modification, so we add one sixth to our running total. Class A was also modified at its fifth-most, fourth-most, and third-most recent commits, so we add that all to the running total, divide by 6 , and end up with a score of .16. Class B has more recent modifications, but less total modifications, but we can see that it still has a higher score at .18 than class A does. Class $\mathrm{C}$ has the least total modifications with only 2 , but it has the highest linear temporal locality score at .25. This exemplifies how heavily the linear temporal locality method favors newer modifications. The logarithmic calculation works very similarly to the linear calculation, but we can see that it penalizes older activity substantially less than the linear method. For class A, we take one divided by $\ln$ of 6 plus one divided by the $\ln$ of 5 plus one divided by the $\ln$ of 4 plus one divided by the $\ln$ of 3 , then divide this all by 6 , producing a score of .47 . Running the numbers in a similar fashion for B, we are left with a score of 0.44 , and 0.33 for class C.

To reinforce these concepts, let us go through a more advanced example. Suppose we have a class, Connector.java, that we want to produce temporal locality scores for as time passes by. When we are given the first ever requirement, no files as of yet 


\section{Table 3.1: Example Of Temporal Locality Result For Three Classes}

\begin{tabular}{|c|c|c|c|c|c|c||c|c|c|}
\hline \multicolumn{1}{|c||}{ Class } & \multicolumn{3}{c||}{ Requirement Touch } & \multicolumn{3}{c|}{ Temporal Locality Results } \\
\cline { 2 - 9 } Name & 1 & 2 & 3 & 4 & 5 & 6 & Simple & Linear & Logarithmic \\
\hline A & X & X & X & X & 0 & 0 & $\mathbf{0 . 6 7}$ & 0.16 & $\mathbf{0 . 4 7}$ \\
\hline B & 0 & 0 & X & X & X & 0 & 0.5 & 0.18 & 0.44 \\
\hline C & 0 & 0 & 0 & 0 & X & X & 0.33 & $\mathbf{0 . 2 5}$ & 0.33 \\
\hline
\end{tabular}

have been modified, so the temporal locality scores are all 0. Suppose then that the class is touched in the first requirement. For the second requirement, we have had a total of one requirement, and that requirement modified the Connector.java class, so each temporal locality score is 1 . Requirement 2 does not modify the class. So now at requirement 3 , the class has been modified $1 / 2$ times for a simple score of .5. For the weighted score, we calculate $1 / 2 / 2$ to arrive at a score of .5. Since our logarithmic algorithm does not start taking logarithms until requirement 3 or greater, the score is also .5 here.

At requirement 4 , we have a history of 3 total requirements, with modifications at requirement 1 and requirement 3 . The simple score is $2 / 3$, the weighted score is $(1 / 3$ $+1) / 3$, and the logarithmic score is $(1 / \ln (3)+1) / 3$. Here, we can already see the contrast between the different algorithms. The older a change is, the less the weighted algorithm places on the importance of the change. The logarithmic algorithm has the same philosophy, but does not penalize older changes quite as much.

At requirement 5, we have a history of 4 total requirements, with modifications at 1,3 , and 4 . The simple score is $3 / 4$, the weighted score is $(1 / 4+1 / 2+1) / 4$. The logarithmic score is $(1 / \ln (4)+1+1) / 4$.

Now let's skip ahead to requirement 8. We have a history of 7 total requirements, with modifications of Connector.java at 1,3 , and 4 . The simple score is $3 / 7$, the 
weighted score is $(1 / 7+1 / 5+1 / 4) / 7$ for a total of .084 , and the logarithmic score is $(1 / \ln (7)+1 / \ln (5)+1 / \ln (4)) / 7$ for a total of .265. Note how much the weighted and logarithmic temporal locality scores drop without any recent modifications.

At requirement 9, we finally have another recent modification. There is a history of 8 total requirements now, with modifications at $1,3,4$, and 8 . The simple score is .5 , the weighted score is $(1 / 8+1 / 6+1 / 5+1) / 8$ for a total of .186 , and the logarithmic score is $(1 / \ln (8)+1 / \ln (6)+1 / \ln (5)+1) / 8$ for a total score of .332 . It is interesting to see how much the logarithmic and weighted scores jump up once there are more recent modifications.

At requirement 14, we have a history of 13 total requirements, with modifications at $1,3,4,8$, and 9 . The simple score has droped to $5 / 13$ for a total score of .3846 , the weighted score is $(1 / 13+1 / 11+1 / 10+1 / 6+1 / 5) / 13$ for a total score of .048 , and the logarithmic score is $(1 / \ln (13)+1 / \ln (11)+1 / \ln (10)+1 / \ln (6)+1 / \ln (5))$ / 13 for a total of .186. Again, note how sharply the temporal locality scores drop when there are no recent modifications.

\subsection{Classifier}

Once all metrics have been calculated and serialized, we create one sample per instance of a touched or untouched class file associated with a RequirementChange. We add in the NLP, static analysis, and temporal locality metrics for each sample, then use this data to train a classifier that makes a simple binary classification - touched or untouched. 


\section{Table 3.2: Temporal Locality Example}

\begin{tabular}{||cccccc||}
\hline Class & ReqId & Previous Modifications & Simple & Weighted & logarithmic \\
\hline \hline Connector.java & 1 & None & 0 & 0 & 0 \\
\hline Connector.java & 2 & 1 & 1 & 1 & 1 \\
\hline Connector.java & 3 & 1 & .5 & .25 & .5 \\
\hline Connector.java & 4 & 1,3 & .67 & .433 & .6366 \\
\hline Connector.java & 5 & $1,3,4$ & .75 & .4375 & .68 \\
\hline Connector.java & 8 & $1,3,4$ & .429 & .084 & .265 \\
\hline Connector.java & 9 & $1,3,4,8$ & .5 & .186 & .332 \\
\hline Connector.java & 14 & $1,3,4,8,9$ & .3846 & .048 & .186 \\
\hline
\end{tabular}

\subsubsection{Feature Pre-processing Configurations}

We employed two different feature pre-processing configurations when training and testing our classifier. They are as follows.

\subsubsection{Principal Component Analysis}

Many models contain features that are either extraneous or highly correlated with other features. Principal Component Analysis, or PCA, is often used to reduce dimensionality in datasets [47]. First Introduced by Pearson, PCA bears many similarities to the process of Singular Value Decomposition in linear algebra. If we consider a matrix, M, which is an $\mathrm{NxP}$ matrix with $\mathrm{N}$ values and $\mathrm{P}$ variables (or features), we can construct a diagonal variable matrix by taking $\mathrm{V}=\mathrm{MM} /(\mathrm{n} 1)$ [47]. The eigenvectors for this data are called the principal directions, and projections onto this space are called the principal components [47]. 
Once PCA is performed, we are left with an approximation of the original matrix that still contains valuable information, but with less variables used. The variables used will often effectively be combinations of variables from the previous matrix M, and should all be independent from one another [70].

We utilized PCA as one of our two feature pre-processing configurations.

\subsubsection{Top 5 Information Gain}

Information Gain can be thought of as the difference in entropy between two states [16]. An attribute with high information gain is likely informative towards a particular class. In machine learning, It is used in several decision tree algorithms as a way to split nodes in the tree-building process.

$$
\begin{gathered}
\text { Entropy }=-p P * \log 2(p P)-p N * \log 2(p N) \\
\text { InformationGain }=\text { EntropyPrior }- \text { Entropy Next }
\end{gathered}
$$

For each dataset, we ran a filter that ranked each feature by its information gain in descending order, then removed all but the top 5 features. This allows us to use our best set of features, eliminating feature sets with low information gain.

\subsubsection{Classifiers}

The following is a brief listing of the classifiers we tested CCP with.

\subsubsection{Naive Bayes}

The Naive Bayes Classifier is a popular classification algorithm commonly used as a baseline for machine learning tasks. It operates on a model that every feature contributes to a classification equally [60]. If an animal is classified as a bear, it could 
have features such as size $=$ large and fur $=$ brown, and each of these features would independently contribute to the classification. After training, each feature is given a probability for a classification called the posterior probability. It then takes the prior probability of the given class if there were 30 bears classified out of 60 animals in the training set, this would be a probability of 0.5 . This is then multiplied by the combined probability of the features for the given class, which forms what is known as the posterior probability (evidence is a constant, and can be ignored for the sake of this explanation) [60]. The class with the highest posterior probability is chosen.

\subsubsection{Bootstrap Aggregation}

Bootstrap aggregation, otherwise known as bagging, is an ensemble classifier, which means that it is an external algorithm that creates additional classifiers based on different parts of the data.

Bootstrap Aggregation consists of two actions: bootstrapping and aggregation. In the bootstrapping portion, the data is sampled repeatedly with replacement. A classifier is then trained on each group of samples. When testing, several classifiers attempt to predict a sample, with the mode classification chosen [23]. The algorithms for bootstrapping and bagging are defined as follows.

- Assume we are splitting the data into $\mathrm{k}$ chunks

- Sample a random chunk of the data

- Sample the rest of the data into $\mathrm{k}-1$ additional chunks, with replacement 


\section{Building multiple classifiers}

for all Chunks from i to $\mathrm{N}$ do

Create a training set from chunk i

Train a classifier on chunk $\mathbf{i}$

end for

Testing

for all samples $\mathrm{j}$ to $\mathrm{A}$ do

Test each classifier on the sample.

The mode classification is chosen.

end for

Algorithm 3: Bagging

\subsubsection{Decision Tree}

A decision tree is is a graph representing various pathways that can be taken, given a set of variables, with a nominal result, or class label, at its leaf nodes [16]. Decision Trees are usually built from the top down, using a breadth-first or depth-first search, placing nodes with the highest information gain towards the top of the tree [16]. To increase the accuracy of the decision tree, most algorithms employ some form of pruning of the tree, effectively removing branches of the tree that are less useful in performing classifications [16].

\subsubsection{The J48 Decision Tree}

The J48 Decision Tree is an implementation of the C4.5 Decision Tree algorithm using the java programming language. The $\mathrm{C} 4.5$ algorithm, like other decision tree algorithms, starts by building a tree from the top down. As the tree is constructed, the tree is split on nodes that yield the highest information gain, which can be thought of as the difference in entropy between the two nodes [71]. Once the tree is finished, 
the top-most nodes from the root should all have one or more branches leading to unique classifications [71]. The algorithm then prunes the tree, putting leaves in place of branches with low usefulness.

\subsubsection{Random Forest}

The Random Forest classifier is an evolution of the decision tree, where a number of separate, randomized decision trees are generated. It is quite similar to the previously introduced bagging algorithm as well, but differs in that when creating decision trees, there is some randomness when choosing what feature to split on. In similar fashion to bagging, once all the trees have been generated, the label with the most consensus among the trees is chosen. This technique has proven to be highly accurate and robust against noise [24]. However, because this classifier requires the building of many different decision trees, it can be extremely expensive to run on large datasets.

\subsubsection{Cross-Validation}

Cross-validation refers to the process of splitting a dataset into $\mathrm{N}$ randomly separated chunks. The chunks are then iterated through; at each iteration, one chunk is held out for testing, while the classifier is trained on the remaining $\mathrm{N}-1$ chunks. The final accuracy metrics are the result of testing all N chunks. Researchers often prefer nfold cross-validation to simple training/test splits, as N-fold validation employs more

rigorous evaluation of the dataset, ensuring that a model less likely to be overfitted $[17]$. 


\section{Chapter 4}

\section{CLASS CHANGE PREDICTOR}

CCP is a complex system consisting of many moving parts. In this chapter, we discuss the various technologies and tools that operate the machinery of CCP. The user must first locate a relevant Git repository and a jira URL for the project. CCP can then be configured to access its data stores and metric providers. Through commandline invocations, CCP then gathers data using the configured values. Finally, CCP outputs a variety of different data files, each of which is used to train a machine learning classifier.

\subsection{Data Stores}

The Class Change Predictor draws from two main data sources in order to perform its predictions on a given software project: the project's code-base, which is historically tracked in a version control system, and the project's issue tracking software, which is tracked via a web-based tool. This combination of textual and technical data allows our system to gain a sophisticated understanding of what situations lead to software changes.

\subsubsection{Jira}

Jira is a software management and issue tracking system commonly used in industry [10]. It is used by commercial tech giants such as Amazon, as well as on opensource Apache projects [10]. Software Development Managers (SDMs) can use Jira to create teams, create projects, and break those projects up into subtasks. Software 


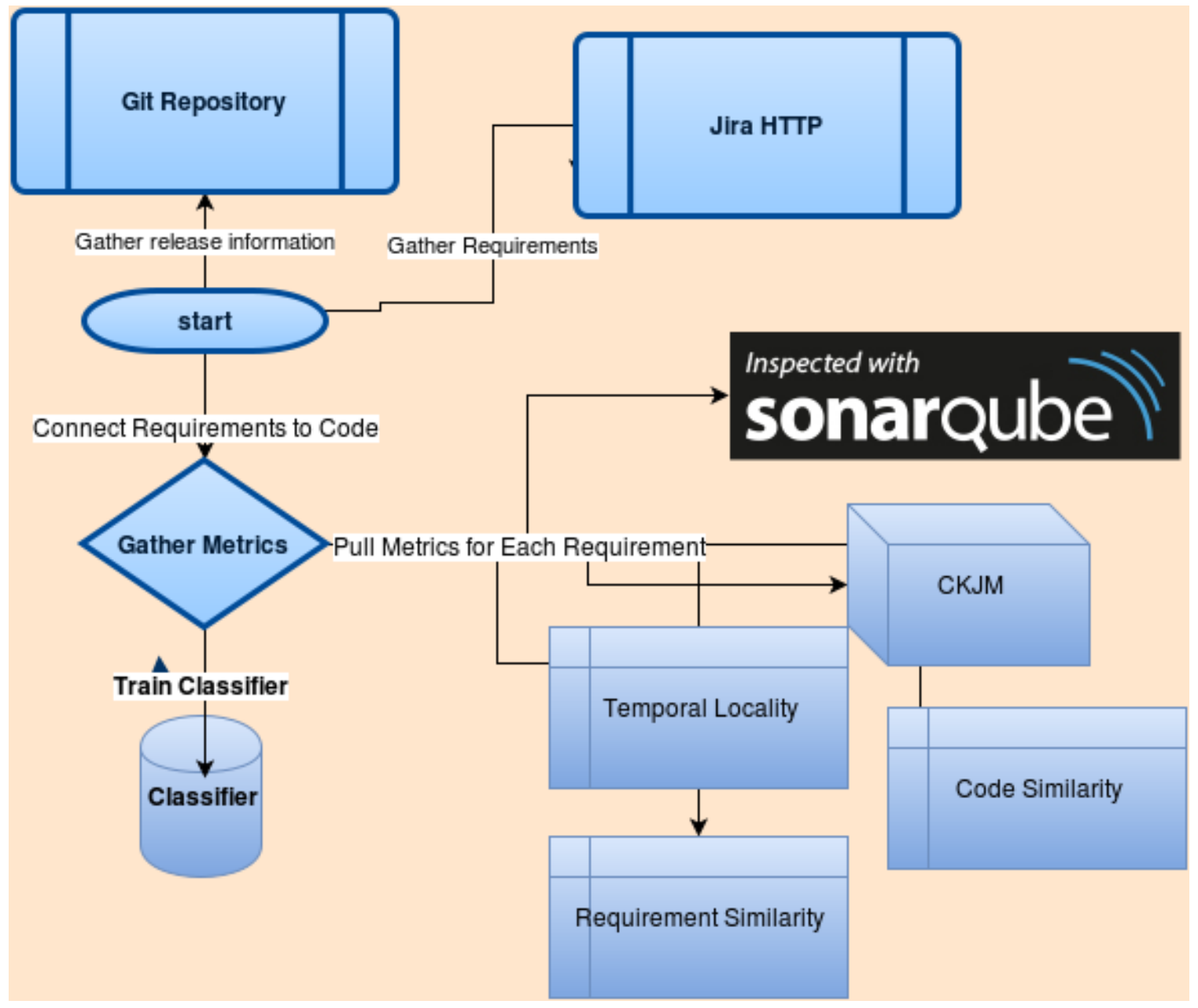

Figure 4.1: System Workflow 
Development Engineers (SDEs) can then take on certain subtasks or create new tasks associated with a specific release.

We chose to focus on Jira systems for our data collection both due to the large amount of openly browsable jira repositories available, as well as the ease of pulling data out of jira with simple JQL queries.

\subsubsection{Jira Extractor}

Large projects usually have thousands of Jira issues, while most open Jira systems usually place a limit on the amount of Jira issues that can be downloaded at a time (typically 100) [10]. Thus, we devised a way to break up our request into several different jira queries, pulling a small number of issues at a time, scraping the HTML into a usable Jira Issue object, and parsing the results into a csv file.

Our Jira data scraping tool finds the first commit date in the repository, and then performs many time delimited jira queries until all issues in the system are collected. The scraper has proven to be robust across many different projects.

1. The scraper takes the first requirement date for a project.

2. The scraper downloads the tickets one hundred at a time, split up by week (no week in any of the repositories had more than 100 tickets in a seven day period).

3. Scraped the HTML list of tickets into a simple CSV format and outputted a final jira CSV file.

\subsubsection{Parsing Jira Data Into Requirements}

Each Jira change request is parsed into a Requirement object. A requirement contains several key fields from the Jira Request, such as 
- The Jira ID of the requirement.

- The summary text of the change request.

- The type of request, such as "Bug", "New Feature", "Improvement", or "Task".

- The release that the requirement is apart of.

- The creation date of the requirement.

Later on in the process, additional fields are added to link a requirement with the state of the code repository at the time.

\subsubsection{Git}

Git is a version control system that is widely used in the software development community [8]. Git users can make changes to a software project, revert changes, make branches for divergent code, and check out specific commits which correspond to the version of a software at a specific point in time. It differentiates itself from SCM tools like SVN in that git allows users to download a copy of the entire repository locally [8].

We chose projects with Git version-control systems due to Git's ubiquity, ease-ofuse, and API support. On a release by release basis, we leverage Git to find and store the state of the code-base. This includes all source code files currently currently in the repository and their associated metadata. This information is stored, to be used at a later time for static analysis and NLP. On a commit by commit basis, we use Git to find what files changed and what files stayed the same. 


\subsubsection{JGit}

We used the JGit API due to its excellent documentation in comparison to other libraries [9]. It is designed to be light-weight, and implements virtually all of Git's core functionality.

\subsubsection{Git Extractor}

Leveraging the JGit API, we created a custom Git Extractor tool. The Git Extractor tool can iterate through all commits, record metadata about the commit, find out what files have changed in the commit, and find out what files have stayed the same in a commit. To facilitate this, we developed several custom methods.

- getAllFiles, which, given a commit as input, returns a list of tuples containing all files currently in the repository, and their creation times.

- getFirstCommitTime, which returns the commit time of the first commit in the repository.

- getChangedFiles, return all files that have changed as a result of the current commit.

- filterFiles, which returns all files in the repository that have been created after a certain time.

\subsection{Build Tools}

Building enterprise-scale projects can often prove a challenging task. Large-scale projects typically depend on many external libraries, require specific build commands and flags, and sometimes use disparate versions of programming languages. As such, 
we developed our system to be robust, with the user able to input custom build commands into the configuration file. CCP has been tested thoroughly using Apache's Maven, but it would also be easy to support other utilities, such as Make, using CCP's XML configuration file.

\subsubsection{Maven}

Maven is a dependency analysis and software package management tool for the java programming language[3]. Developing robust java programs often requires downloading many external java packages that might depend on very specific versions of each other. Developers use Maven to ease the pain involved in this process; developers can specify a central package, and then Maven constructs a dependency graph, downloading and building all additional necessary packages.

For Java projects, we preferred to build with the Apache Maven tool, as Maven can analyze a project's dependencies, download all of the needed libraries from maven's central database, and then build the project [3]. As Maven is itself an Apache project, almost all of the Apache software projects we surveyed were built using Maven.

Both SonarQube and CKJM require compiled bytecode to generate complete metrics for Java classes, so Maven's ability to ease the build process of complex projects is critical.

\subsection{Release Metrics Storage And Tools}

CCP gathers CKJM and SonarQube metrics static analysis metrics at the point

of each software release. This release data is stored in a data structure consisting of a series of nested hashmaps, indexed by release, class name, and metric name 


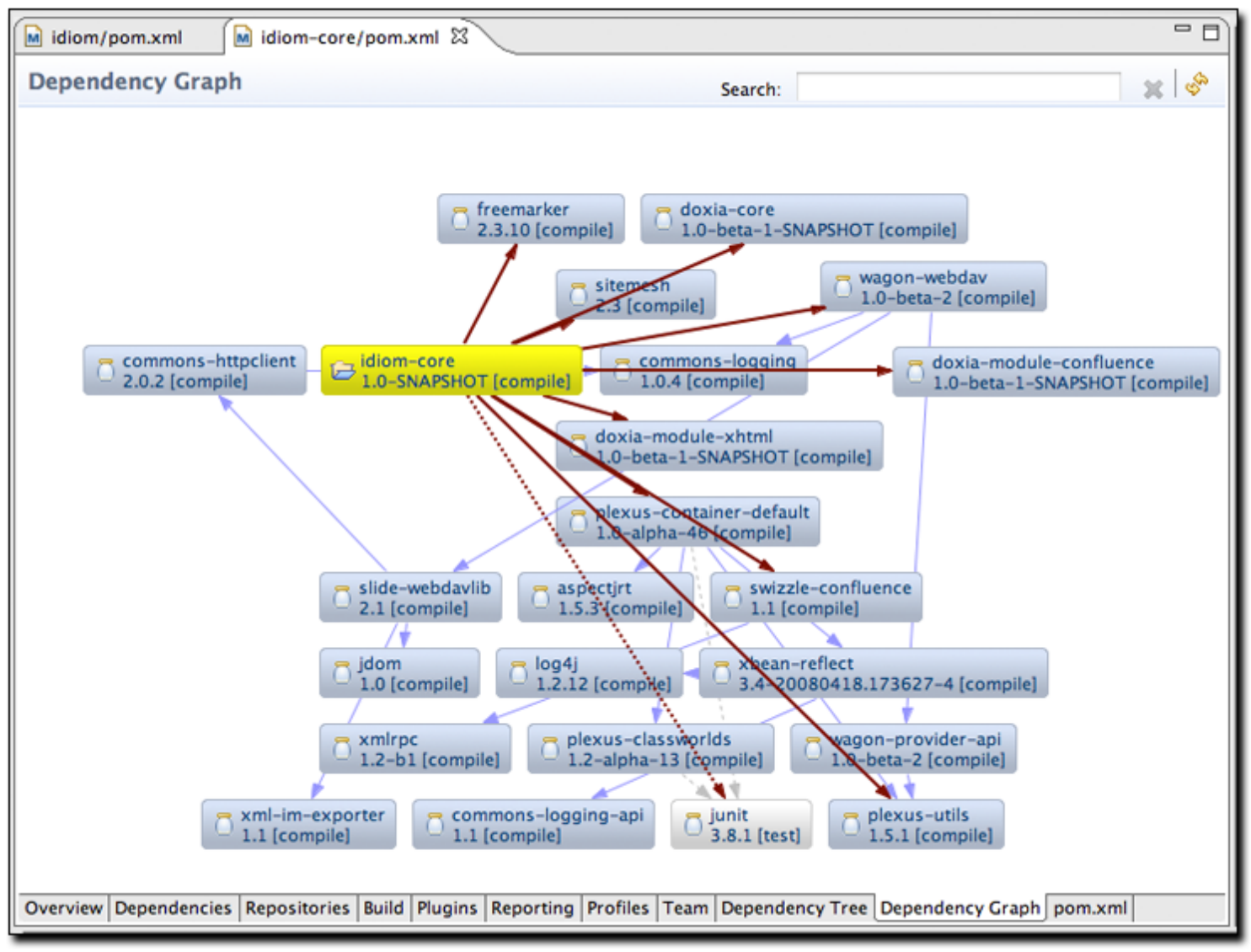

Figure 4.2: A Maven Dependency Graph 


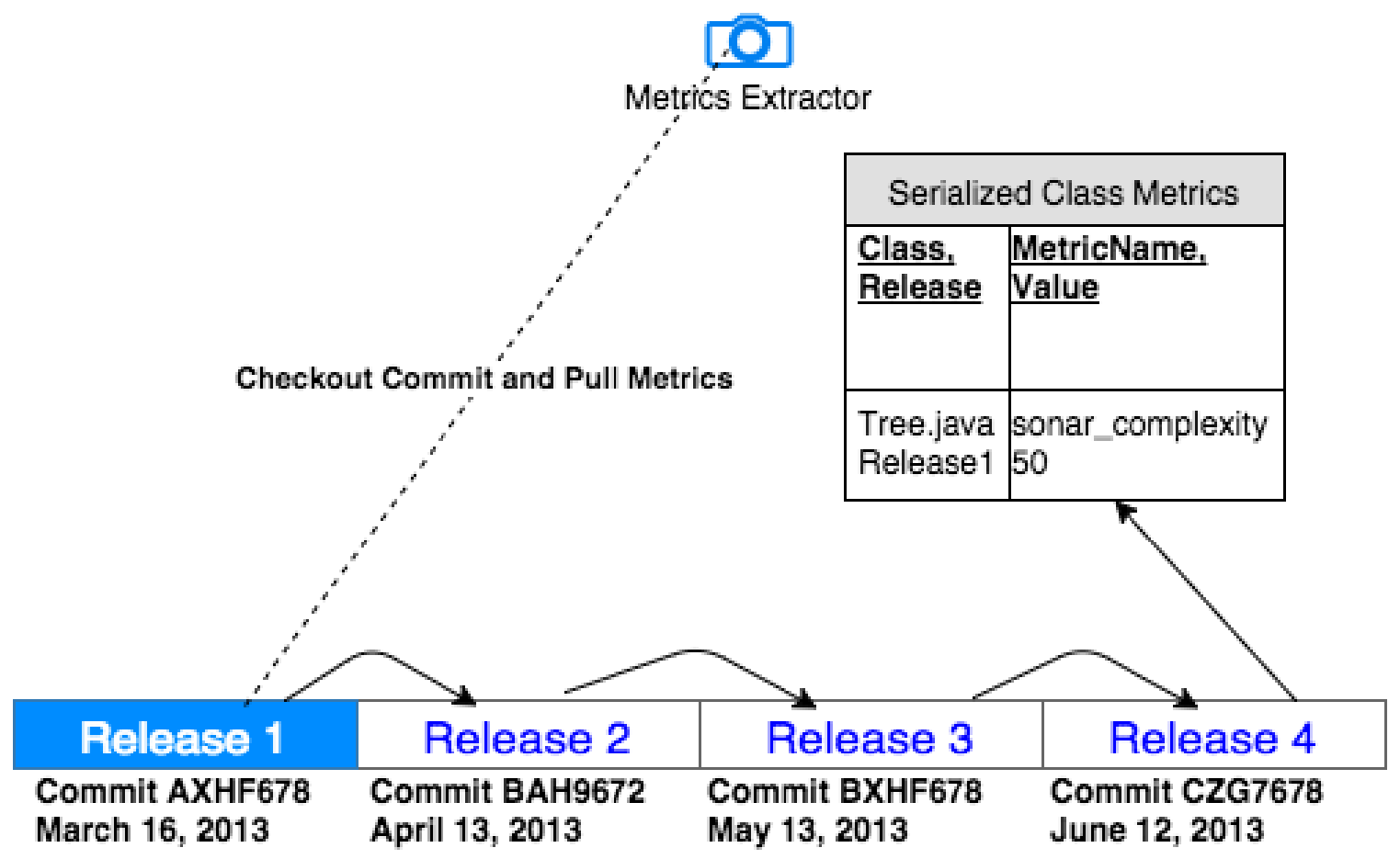

\title{
Figure 4.3: How The System Snapshots Releases
}

respectively. We have constructed several tools to facilitate interacting with these two systems and extracting metric data.

\subsubsection{SonarQube}

SonarQube is a software analysis tool that can track metrics such as technical debt, code smells, and lines of code [67]. It is commonly used by software development teams to track the evolution of code quality over time [67]. It is configured with

\author{
Map of Release String to \\ Map of Class String to \\ Map of Metric String to \\ Numeric metric Value
}

Figure 4.4: Map Of Metric Storage Structure 
a variety of quality rules out-of-the-box, and developers can create unique rules at will. Though we primarily used SonarQube to extract static analysis metrics, CCP could be configured to gather dozens of other metrics from SonarQube on a per class basis. For this reason, we chose SonarQube over simpler static analysis tools that just compute the number of code smells, such as FindBugs.

\subsubsection{SonarQube Executor}

Once all releases (and their corresponding names and commit ids) have been extracted from the project, the SonarQube executor is executed for every release. To ensure the quality and correctness of the SonarQube data, we delete all existing data for a project from the SonarQube database before checking out a release. Once a release is

checked out, the SonarQube Executor executes the Sonar Runner service to analyze the current state of the given software project. This often takes several minutes, depending on the size of the project. There is also usually some delay between when SonarRunner finishes and when the project data is actually available for API calls. Because of this, we developed the SonarQube executor to perform periodic HTTP requests to confirm the project's readiness for API calls. Once the SonarQube Executor receives a successful confirmation, it signals to the SonarQube Scraper that data is ready for analysis.

\subsubsection{SonarQube Scraper}

We built a robust tool to facilitate the extraction of SonarQube metrics from large software projects. Our tool pulls data from SonarQube via HTTP Get commands with the JSON option specified to be true. We parse the JSON metric data into HashMaps, using the name of the class as a key to another HashMap with filenames 
as keys and floating point numbers as values. All of the computed metric data is then serialized to a file for later use.

\subsubsection{CKJM Extractor}

We first modified the open-source CKJM project to expose the ClassMetrics class, as well as methods to gather all CKJM data without resorting to command-line calls [68]. We then removed the command-line interface to the project, and added our own method to parse all CKJM metrics for all compiled classes in a project. We compiled all of this into a JAR file and included it in our CCP project. Given a directory, our modified CKJM JAR will look for all .class files and compute the metrics for each separate class, and then look for any .jar files and compute the CKJM metrics for all the classes therein. Because CKJM requires java bytecode files, CCP only gathers CKJM metrics for java development projects.

When a release has been checked out and the target project has finished building, the CKJM extractor runs, extracting CKJM data for each class file, and storing this data inside of the Release Metrics hash map.

\subsection{Other Technologies}

\subsubsection{The Semantic Similarity Toolkit}

We made use of the Semantic Similarity Software toolkit, otherwise known as Semilar, in order to produce similarity scores between text. We chose this tool because of maturity of its API - it provides a variety of different ways to determine similarity between sets of text. More information on the various similarity calculations used can be found in the approach section. 


\subsubsection{Weka}

The Weka library is a machine learning toolkit developed by the University of Waikato [12]. We made use of the Weka machine learning library due to its ease of use, wide array of classifiers, and robust pre-processing tools[12]. Weka contains a total of 83 classifiers and at least 50 processing utilities. Using our system, users can train a JF48 classifier and test its accuracy via a command-line call.

\subsubsection{Attribute-Relation File Format}

The Attribute-Relation File Format, or ARFF, was designed for use with Weka by the the University of Waikato. ARFF files consist of header and data sections. The header section, denoted with @Header, contains the names of each attribute used in the file. The data section, denoted with @Data, contains all rows of data, called instances. By convention, the class label is typically placed as the last of the data's attributes. It is a convenient, human-readable format in which to store training data.

Once CCP has completed processing necessary requirement, commit, and static analysis data, it processes this information into several ARFF files. One file is created for each feature, each feature group, and all of the combined features. In addition, CCP performs PCA and generates additional PCA files for each feature group, as well as for all of the combined features.

\subsection{Configuration And Operation}

In order to support a variety of different types of projects, we made the CCP tool extremely customizable by utilizing an XML configuration file, as well as providing the ability to use command-line arguments. 
Table 4.1: Configuration Options

\begin{tabular}{|c|c|}
\hline Option & Purpose \\
\hline git_repo & The location of the Git repository to be analyzed. \\
\hline jira_file & The file name of Jira file to store requirements data in. \\
\hline jira_url & The URL of the jira repository that will be queried. \\
\hline req_limit (Optional) & $\begin{array}{l}\text { The number of requirements to process before exiting } \\
\text { the linking phase. Useful for testing. }\end{array}$ \\
\hline jira_url & The URL of the jira repository that will be queried. \\
\hline statics_file & $\begin{array}{l}\text { The location on disk of the serialized file that holds } \\
\text { static metrics. }\end{array}$ \\
\hline sonar_command & Command used to run SonarQube on a project. \\
\hline sonar_host & URL of the SonarQube server to extract data from. \\
\hline sonar_project & $\begin{array}{l}\text { Project name to use for the sonar repository. Defaults } \\
\text { to the name of the git repo. }\end{array}$ \\
\hline sonar_metrics & $\begin{array}{l}\text { Comma-separated list of metrics to extract for each class } \\
\text { from SonarQube. }\end{array}$ \\
\hline
\end{tabular}

\subsubsection{Configuration}

A variety of configuration options are available, the majority of which are required for operation of the tool. A table listing the available configuration options, as well as an example XML configuration file, are provided.

\subsubsection{Commandline Operation}

A variety of commandline flags and options are provided to ease the operational burden for users of CCP. 
Table 4.2: Command-line Flags

\begin{tabular}{|c|c|}
\hline Flag & Use \\
\hline config & $\begin{array}{l}\text { Used to specify the XML configuration file to load settings from. } \\
\text { Mandatory }\end{array}$ \\
\hline jira & Run the Jira Scraping utility, then serialize the results to disk. \\
\hline reqnum & $\begin{array}{l}\text { Similar to the XML option, specify the number of requirements to } \\
\text { process. }\end{array}$ \\
\hline reqs & $\begin{array}{l}\text { Process all requirements, link them to commits, and serialize the } \\
\text { requirements to disk. }\end{array}$ \\
\hline statics & Run static analysis on every release, serializing the results to disk. \\
\hline bigtable & Generate a single table file with all combined metrics. \\
\hline split & $\begin{array}{l}\text { Used to re-organize a training and test so that "perfect" require- } \\
\text { ments appear at the beginning of a file }\end{array}$ \\
\hline $\operatorname{arff}$ & $\begin{array}{l}\text { Used to indicate the need to dump all data to weka-formatted } \\
\text { ARFFs, split up by feature groups. }\end{array}$ \\
\hline exclude_missing & When processing data, filter out lines with missing metric values \\
\hline delete_untouched & $\begin{array}{l}\text { Remove requirements from the dataset that aren't associated with } \\
\text { any touched java or php files. }\end{array}$ \\
\hline join & $\begin{array}{l}\text { When the user specifies a comma seperated list of serialized metrics, } \\
\text { this flag is used to join all the metrics into one file. }\end{array}$ \\
\hline language & $\begin{array}{l}\text { Used to specify the programming language used in the project. } \\
\text { Defaults to Java. }\end{array}$ \\
\hline report_releases & Output a report with release statistics. \\
\hline report_requirements & Output a report with requirement statistics. \\
\hline
\end{tabular}




\subsection{Overall Process}

The typical workflow of the CCP system is listed below. This is by no means comprehensive, as it omits the use of several non-essential pre-processing capabilities

1. The user must first clone the target project Git Repository (manual).

2. The user then prepares project configuration file (manual).

3. The user then runs CCP.

4. CCP Iterates through the list of requirements. Each ticket is associated with one or more commits from the repository. A list of formatted requirements is serialized into multiple files.

5. CCP prepares a list of releases for the project. To figure out the commit hash for each release, we take the first ticket to implement the release, then take its parent. We are technically using the parent of each release, but this makes some sense. We want the state of the code just before the release in order to accurately predict what the release will implement.

6. For each release, CCP checks out the commit for that release, builds the project, runs sonarqube for that project, runs ckjm, and extracts all the metrics.

7. After an outside source computes requirement to requirement and code to requirement semantic similarity metrics, CCP incorporates these metrics into each line of the final file.

8. CCP parses the combined data for each class into ARFF files required by Weka. Two ARFF files are produced for each metric group: one with PCA performed, 
and one without PCA performed. Split each individual metric into its own file. Finally, CCP outputs two files for all of the combined metrics: one with PCA performed, and one without PCA performed.

9. CCP then analyzes, trains, and tests the ARFF files using Weka with tenfold cross-validation. This step can also be performed by a human manually using the weka explorer interface.

\subsubsection{Operation Scenarios}

Due to the wide variety of command-line and configuration options, the operation of CCP may seem daunting. To aid in the use and understanding of CCP, some common operation scenarios are provided below.

\subsubsection{Scenario 1: Compute And Serialize Requirements}

In our first scenario, a user has configured an xml file called "tika.xml" and filled in all appropriate fields. She wishes to run the jira scraper tool, parse those jira issues into requirements, link all the requirements to commits, and output a text file with a list of all serialized requirements. She executes the following command.

- config tika.xml jira reqs

CCP then executes the jira scraper tool and the requirement parsing tool, outputting several files, among them:

- A file listing each class found in the target software project.

- A file listing all of the requirement text found in the software project

- A file listing all releases in the project, and its associated commit ID. 
- A file listing all requirements in the software project, which links to previous files.

- A file listing all issues tagged as Bug fixes in the software project.

\subsubsection{Scenario 2: Analyze Releases}

The user wishes to perform static analysis on each release. The user wishes to link the requirement change data and the associated metrics for each file all together in one output table. She runs the following command.

-config tika.xml statics bigtable

In sequence, CCP runs static analysis for each release, then runs the "big table" routine, which links all available metrics together inside one file.

\subsubsection{Scenario 3: Prepare ARFF Files}

The user wishes to generate ARFF files to be used to train Weka machine learning classifiers. She runs the following command.

-config tika.xml arff

CCP analyzes the "big table" file generated earlier, then generates ARFF files for this table, as well as for each group of metrics in the table, and each individual metric in the table. A total of 44 different ARFF files are generated.

\subsubsection{Scenario 4: Ensemble}

The user wishes to run all the previous steps again, in order. She executes the following command.

-config tika.xml jira reqs statics bigtable arff 
CCP runs all of the previous steps, in order. CCP outputs several requirementsrelated files, a file representing the serialized static analysis files, and at least 44 different ARFF files. 
Chapter 5

\section{EVALUATION}

To evaluate the system, we used CCP to gather datasets from four different projects of varying sizes. We performed one-hundred times ten-fold validation using seven different classifiers and two different feature configurations.

\section{$5.1 \quad$ Variables}

For our experiments on the three java projects, we utilized 34 different independent variables as well as three different dependent variables. A brief summary of these variables follows.

\subsubsection{Dependent}

For our dependent variables, we ran our experiments and the results using the dependent variables precision, recall, and 0.5F-Measure. The reasoning behind each dependent variable is as follows.

\subsubsection{Precision}

In terms of our system, we measure true positives (TP), false positives (FP), true negatives $(\mathrm{TN})$, and false negatives $(\mathrm{FN})$ as follows.

- TP: A class file is successfully predicted to have changed.

- FP: A class file is unsuccessfully predicted to have changed.

- TN: A class is successfully predicted to remain unchanged. 
- FN: A class is predicted to remain untouched when it actually changed.

Precision is a measure of true positives divided by the number of false positives added with true positives. For the CCP system, it is imperative that the system maximizes true positives while minimizing false positives. A true positive indicates that a class has changed. Incorrectly classifying a class that needs to be changed could lead to wasted effort by a developer, including but not limited to a developer refactoring a class that does not need to be touched. We thus weight the importance of precision as a dependent variable higher than recall, as precision penalizes false positives, while recall penalizes false negatives.

$$
\text { Precision }=\frac{T P}{F P+T P}
$$

\subsubsection{Recall}

Recall measures the number of true positives divided by the number of true positives added with the numbers of false negatives. Recall has a major weakness in that a classifier choosing every entry of positive will have a recall of one; recall does not penalize false positives.

$$
\text { Recall }=\frac{T P}{T P+F N}
$$

\subsubsection{F 0.1 Score}

Even though precision is objectively the most important measure for the CCP system, F scores also provide valuable evaluation data. The F0.1 score combines both precision and recall in to one averaged score. While the traditional F1 score takes the harmonic mean between precision and recall, the F0.1 score places much higher emphasis on precision by lowering the Beta multiplier in the below formula. For our 
approach, this makes sense, as we believe that a FP costs more development effort than a FN.

$$
\begin{gathered}
F b=\left(1+b^{2}\right) * \frac{\text { precision } * \text { recall }}{\left(b^{2} * \text { precision }\right)+\text { recall }} \\
F 0.1=(1+.01) * \frac{\text { precision } * \text { recall }}{(0.01 * \text { precision })+\text { recall }}
\end{gathered}
$$

\subsubsection{Independent}

We employed the use of 34 different independent variables, each representing a feature or metric in our machine learning classifier.

\subsubsection{SonarQube Violations}

The Sonarqube java plugin used in this project comes with over 1000 rules setup [67]. The rules can be code-smell related, such as "private methods that do not access instance data should be static", performance-related, such as "'wait(...)' should be

used instead of "Thread.sleep(...)" when a lock is held", and security related, such as "'public static' fields should be constant". We hypothesize that if a class violates several of these rules, it is likely to need refactoring, and thus more likely to be changed. For each class file in a release, we measure the number of code quality rule violations.

\subsubsection{SonarQube Code Complexity}

Code complexity in Sonarqube is a simple measure of the number of key-words (if, else, for, while, etc.) used in a code fragment [67]. 
We hypothesize that the more complex a class file is, the more likely it will be to need refactoring. We thus take a measure of the code quality for each class in a release.

\subsubsection{Lines Of Code}

We hypothesize that larger class files are more likely to be in need of refactoring. We measure the number of lines of code (excluding white space and curly braces) for each class file in each release.

\subsubsection{Change Frequency}

The change history metric is simply a class's number of past touches divided by its total touch opportunities. We hypothesize that in many software projects, class files changed in the past are likely to be changed again in the future.

\subsubsection{Linear Temporal Locality}

We hypothesize that in many code-bases, classes that have been modified more recently are more likely to change in the future. We employ linear temporal locality scores, which heavily weight more recent changes to class files, to each class based on recent modification history.

\subsubsection{Logarithmic Temporal Locality}

Logarithmic temporal locality, like the linear variety, weights recent modifications more than "stale" modifications. However, it penalizes "stale" modifications much less than the linear metric. We hypothesize that the logarithmic metric will be an effective balance between the previous two approaches. 


\subsubsection{CKJM WMC: Weighted Methods Per Class}

The WMC is the sum of the cyclocomatic complexities for each method in a class [28]. If a class has 3 methods, with method foo having a complexity of 3 , method bar having a complexity of 5 , and method fizz having a complexity of 2 , the WMC of the class will be 10 . We hypothesize that classes with complex methods are more likely to be refactored.

\subsubsection{CKJM DIT: Depth Of Inheritance Tree}

The DIT measures the number of levels in a class's inheritance tree. If one class extends another class in Java, the child class will possess the methods of the parent class. Thus, the DIT measure shows how much code re-use a class has [28]. We hypothesize that classes with higher DIT scores are less likely to be refactored.

\subsubsection{CKJM NOC: Number Of Children}

The NOC score measures the number of times a given class has been sub-classed. Sometimes in industry, a class can be implemented with poor abstraction, and its children need to make many custom member variables as a result, leading to more total sub-classes in the project [28]. It is our hypothesis that classes with higher NOC scores are likely in need of refactoring, and thus more likely to be modified by the user.

\subsubsection{CKJM CBO: Coupling Between Object Classes}

For each class-file, CBO measures the number of non-inheritance related connections to other classes. Classes with a high CBO measure are generally much harder to modify, as modifications to the class in question can affect many other class files [28]. 
We hypothesize that classes with high CBO are likely in need of refactoring, and thus more likely to be modified.

\subsubsection{CKJM RFC: Response For A Class}

The RFC is the count of all methods that can be invoked by a class in response to user input. A high RFC score is indicative of a class that will be difficult for developers to understand, and thus more time-consuming to modify. We hypothesize that classes with high RFC scores are likely in need of refactoring, and thus more likely to be modified.

\subsubsection{CKJM LCOM: Lack Of Cohesion In Methods}

The LCOM score is used to measure the level of cohesion of methods in a class. If a class has many methods that operate on the same instance variables, it will have high cohesion. If a class has multiple methods that have no instance variables in common, it will have low cohesion [28]. We hypothesize that classes with low LCOM scores are likely in need of refactoring, and thus more likely to be modified.

\subsubsection{CKJM Ca: Afferent Couplings}

For a given class, its afferent coupling score measures the amount of times that other classes in the project reference the given class. Classes with high afferent coupling scores are usually more difficult to modify, as sometimes many other coupled classes will need to be modified along with it [28]. We hypothesize that classes with high afferent coupling scores are likely in need of refactoring, and thus more likely to be modified. 


\subsubsection{CKJM NPM: Number Of Public Methods}

NPM in java measures the number of methods that have been exposed as public. Classes with many public methods can sometimes have poor cohesion, given that any accessing class can call the class's methods [28]. We hypothesize that classes with low LCOM scores are likely in need of refactoring, and thus more likely to be modified.

\subsection{Projects}

Generally, the more (and more varied) the data, the more accurate and robust the classifier [34]. Thus, we sought to prioritize projects with higher number of commits and requirements. For each project, we examined how many requirements were available in the project Jira as well as how many commits were available in the project's Git repository. Using a heuristic that projects with higher link ratios (the ratio of requirements that can be linked to a commit in the repo vs. the total number of requirements) would be higher quality projects, we developed a formula that takes all of the mentioned heuristics into account. We computed a total score for the top eight projects in terms of link percentage, and then chose the top four projects for analysis.

$$
\text { Suitability }_{S} \text { core }=(\text { Requirements } * \text { Link } \%)+0.3 * \text { Commits }
$$

A sixth industrial project was provided to us by the California State Polytechnic University in San Luis Obispo [6].

In the case of the apache java projects, a valid requirement constitutes a requirement that contains a source code modification to a java file. In the case of digital democracy, a valid requirement constitutes a requirement that contains a source code modification to a php file. '\%Code' column represents the percentage of lines of code 
Table 5.1: Statistics For The 4 Datasets

\begin{tabular}{|l|c|c|c|c|c|}
\hline \multirow{2}{*}{ Project } & \multicolumn{2}{|c|}{ Requirements } & Commits & Link\% & \% Code \\
\cline { 2 - 5 } & Linked & Valid & & & \\
\hline accumulo & 747 & 577 & 7889 & 64 & 86.2 \\
\hline digital & 59 & 27 & 1242 & 25 & 14.8 \\
democracy & & & & & \\
\hline tika & 231 & 187 & 2781 & 70 & 91.9 \\
\hline isis & 667 & 624 & 6521 & 63 & 94.7 \\
\hline
\end{tabular}

in the project that match our target for the project; so for the java projects, the '\%Code' column represents the percentage of lines of code that are written in java, and for digital democracy, '\%Code' represents the percentage of lines written in PHP.

\subsubsection{Selected Projects}

\subsubsection{Apache Tika}

Apache Tika is an open-source development project dedicated to parsing a variety of different file-types and extracting their metadata [4]. It has been in development since June of 2007, with its first major release in December of the same year. Though on first glance, Tika has over one thousand requirements, we found that a large amount of them were unusable, either due to not containing java files in the commit, requirements did not include a release, or because several releases could not be compiled using Maven. Though it has a relatively small number of usable requirements and commits, we sought to include at least one apache project with a smaller dataset. 


\subsubsection{Apache Isis}

Apache Isis is a framework made in java to be used for rapid web development. It features a ready-made UI component as well as easy to use RESTful services [2]. Its extremely high number of usable requirements make it an ideal subject for our experiments.

\subsubsection{Apache Accumulo}

Apache Accumulo is a distributed key-value database system that bears some similarity to Amazon's DynamoDB [1]. It features an extremely large code-base, as well as a high number of usable requirements.

\subsubsection{Digital Democracy}

Unlike the other systems, Digital Democracy is a closed-source PHP project. Because it is a PHP project, the CKJM metrics were unavailable for it, as they require compiled java byte code [6]. Though the Digital Democracy dataset contains a small number of requirements and commits, it is useful to chart system performance on smaller datasets. In contrast to the other systems, we linked the requirements to code by hand in digital democracy's dataset.

\section{$5.3 \quad$ Results}

Our experimental results are as follows. 


\subsubsection{RQ1: What Is The Best Configuration Of Our Approach?}

In our first experiment, we evaluated three different feature configurations. They are as follows.

- Principal Component Analysis

- Top Five Features by Information Gain

We trained and tested six different classifiers using each feature of the three feature configurations. We also tested with a ZeroR classifier, which simply predicts the mode class (negative in our case) every time. The list of classifiers is as follows.

- ZeroR

- Random Forest

- Naive Bayes

- Logistic

- J48

- BayesNet

- Bagging

Once our experiments were completed, we recorded the TP, FP, TN, and FN values for each dataset. We then used these cumulative values to calculate the weighted average precision, recall, and $0.1 \mathrm{~F}$ score for each classifier/configuration combination. 


\begin{tabular}{|c|c|c|c|c|}
\hline Classifier & Pre-processing & Precision & Recall & F0.1 \\
\hline \multirow[b]{2}{*}{ Bagging } & PCA & 0.81 & 0.31 & 0.8 \\
\hline & Top5 & 0.75 & 0.5 & 0.74 \\
\hline \multirow[b]{2}{*}{ BayesNet } & PCA & 0.21 & 0.39 & 0.21 \\
\hline & Top5 & 0.48 & 0.55 & 0.48 \\
\hline \multirow[b]{2}{*}{ J48 } & PCA & 0.75 & 0.34 & 0.74 \\
\hline & Top5 & 0.72 & 0.49 & 0.72 \\
\hline \multirow[b]{2}{*}{ Logistic } & PCA & 0.39 & 0.03 & 0.32 \\
\hline & Top5 & 0.16 & 0.02 & 0.14 \\
\hline \multirow[b]{2}{*}{ NaiveBayes } & PCA & 0.13 & 0.19 & 0.13 \\
\hline & Top5 & 0.21 & 0.12 & 0.21 \\
\hline \multirow[b]{2}{*}{ RandomForest } & PCA & 0.77 & 0.35 & 0.76 \\
\hline & Top5 & 0.71 & 0.55 & 0.7 \\
\hline
\end{tabular}

Figure 5.1: Configuration Comparison

\subsubsection{Best Configuration}

To evaluate our best feature configuration, we trained all seven of our chosen classifiers with each of the given configurations. Overall, the set of "top 5" information gain ranked features was our highest-performing feature set, with an average F0.5 score of 0.4995 and a high score of 0.696 .

\subsubsection{Best Pre-processing Configuration}

To evaluate our best feature pre-processing configuration, we trained all seven of our chosen classifiers with each of the given configurations. Overall, the set of features with PCA applied was our best-performing feature set, with a high F0.1 score of .80 when combined with the Bagging classifier. In fact, the datasets with PCA applied performed better than top5 information gain on four out of the six classifiers. This shows that more than five of our features are useful in most classification tasks. 


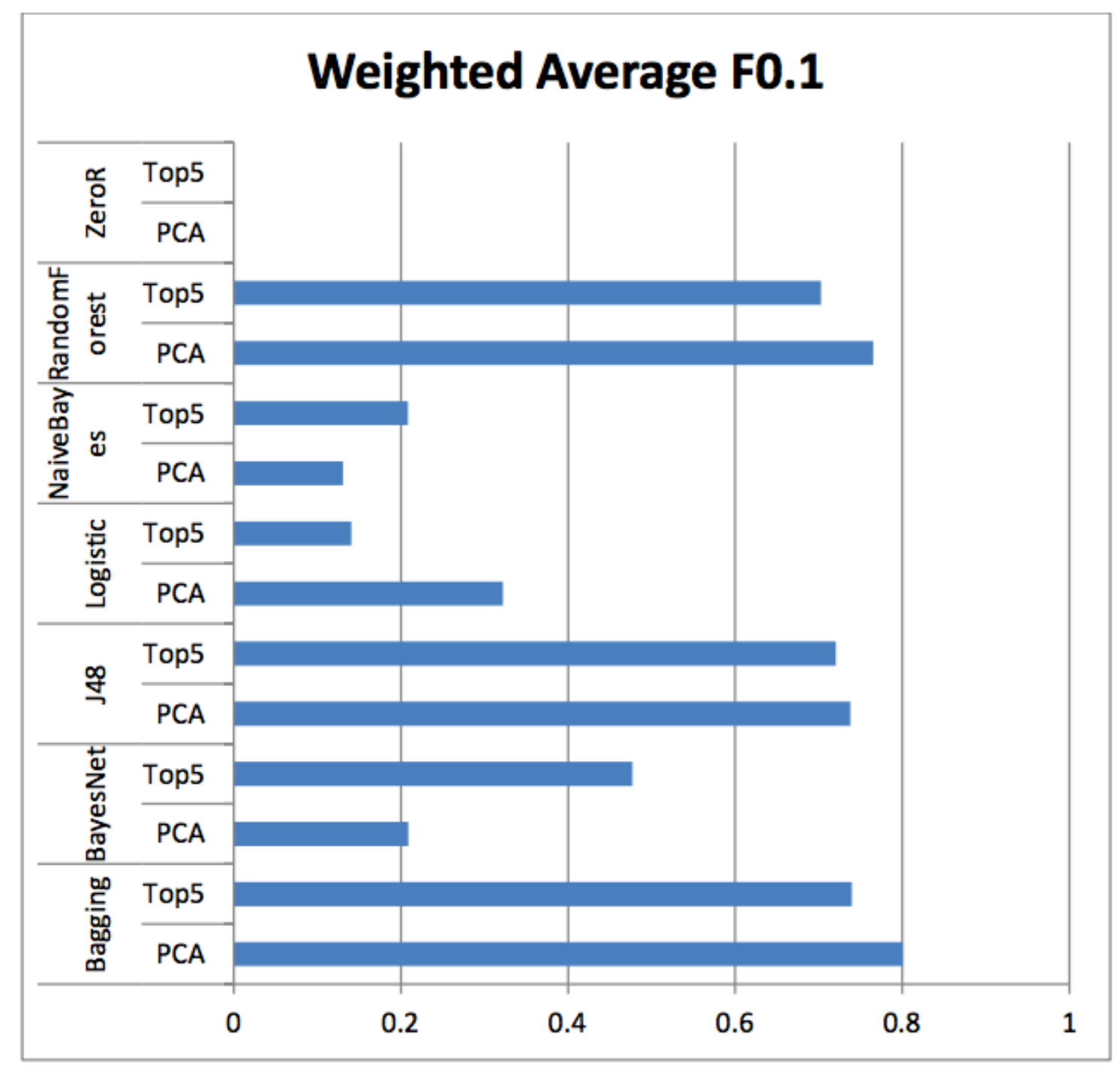

Figure 5.2: Configuration Table

Table 5.2: Highest F0.5 Scores By Feature And Classifier

\begin{tabular}{||ccc||}
\hline Feature Configuration & Classifier & F0.5 Score \\
\hline \hline PCA & Bagging & .80 \\
\hline Top5 & Bagging & .74 \\
\hline
\end{tabular}




\subsubsection{Best Classifier Configuration}

Our best performing classifier across all datasets in terms of average weighted F0.1 score was Weka's bagging classifier, followed by the Random Forest classifier. Both are ensemble classifiers that sample the data many times, with our bagging classifier set to perform ten iterations, and our random forest classifier set to build one hundred trees per sample. They classifiers prioritize accuracy over run-time, so they can be reasonably expected to perform better than the other classifiers.

The J48 classifier performed fairly closely with Random Forest. This is unusual, as the J48 tree is an implementation of the $\mathrm{C} 4.5$ algorithm, and creates just a single decision tree. Note that the ensemble classifiers by default use Weka's custom RepTree "fast decision tree" algorithm, which only sorts numerical attributes once. So it is likely that the J48 classifier is generally more accurate than RepTree, and configuring the ensemble classifiers with J48 would yield even higher f0.1 scores.

The next best classifier was the Bayesian Network classifier, with a .48 F0.1 score. From here, there was a severe dropoff in accuracy, with the Logistic and Naive Bayes classier at .21 and .32 respectively. For Naive Bayes, this was expected, as it is used more as a base-line classifier. We used the ridge estimator when configuring the Logistic Regression classifier; it is possible that using another estimator, such as the lasso estimator, might increase accuracy slightly.

\subsection{RQ2: What Was The Overall Accuracy Of Our Approach?}

For each dataset, we evaluated the performance of our best configured classifier Bagging with PCA - using one hundred times ten-fold cross-validation.

We achieved by our best results on the Accumulo, Isis, and Tika datasets. The Isis and Accumulo datasets contained several orders of magnitude more data than 
Table 5.3: Accuracy Per Dataset

\begin{tabular}{||cccc||}
\hline Dataset & Precision & Recall & F0.1 Score \\
\hline \hline Accumulo & .76 & .30 & .75 \\
\hline Digital-democracy & 0 & 0 & 0 \\
\hline Isis & 89 & .33 & .87 \\
\hline Tika & .78 & .21 & .76 \\
\hline
\end{tabular}

the other two datasets, at 1294411 and 167659 usable samples respectively. This lends credence to our hypothesis that the more training data we have available, the more accurate the classifier will be. Most notably, the Bagging classifiers for Isis and Accumulo had extremely high precision, at .76 and .89 respectively, while not sacrificing much in the way of recall. When these classifiers predicted a source file to change, the prediction was usually correct.

The Tika dataset, while smaller than the previous two, still had reasonable precision and F0.1 score, at .78 and .76 respectively. However, the Tika classifier often produced falsed negatives, with a low recall rate of .21.

However, the DigitalDemocracy dataset proved impossible for our bagging classifier to predict, with 0s across the board. We found that the classifiers built on this dataset predicted untouched classes almost every time. Again, this can be partly be attributed to the small sample size of the data, as it contained only 14 positives compared to 1088 negatives. Oftentimes requirements in this dataset would result in modifications to HTML, CSS, and Javascript files rather than PHP files; in fact, only $50 \%$ of the commits in the dataset included modifications to PHP files. This makes it more difficult to capture a traceable dataset. As well, we were unable to gather CKJM complexity metrics, as there is no open-source plugin to gather them in the 


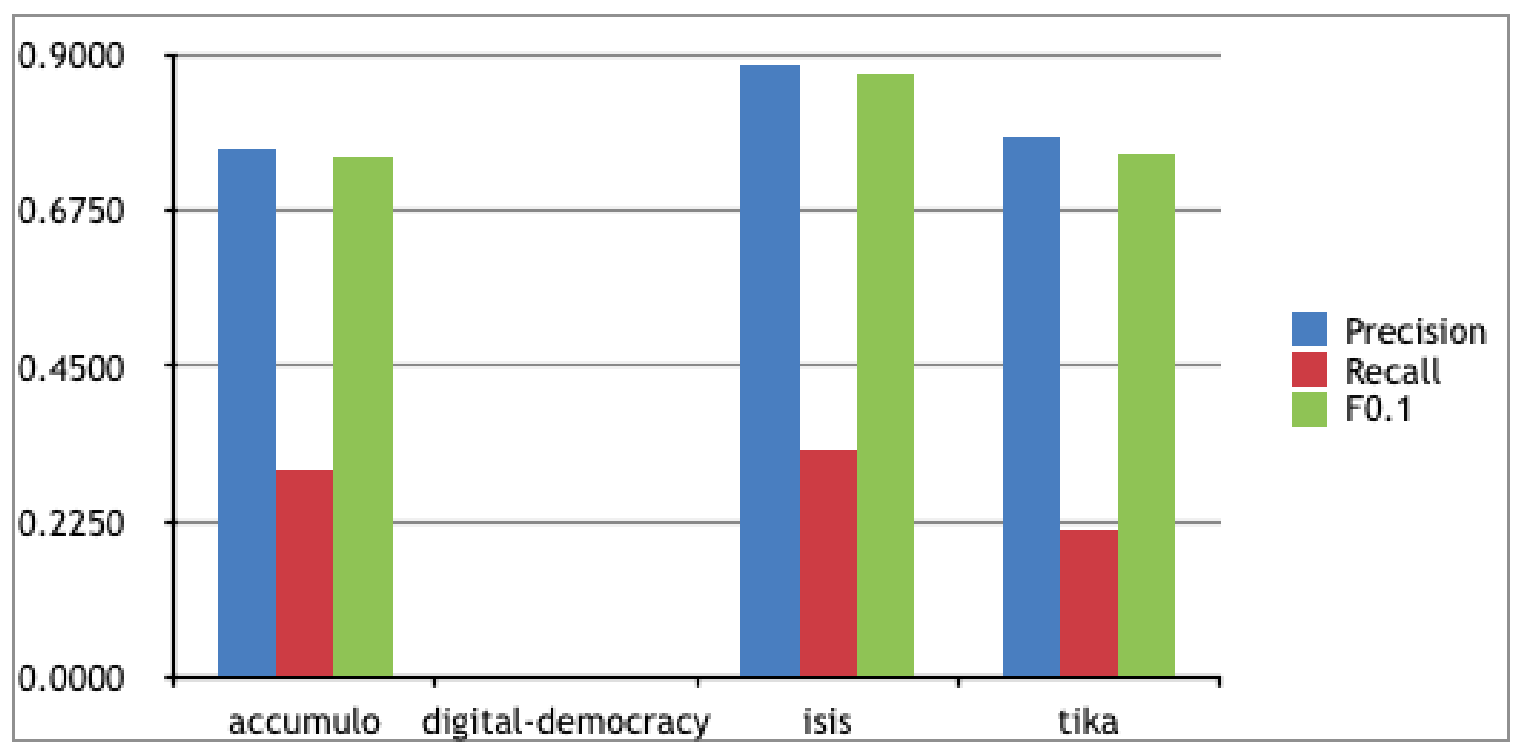

Figure 5.3: Evaluation Data Across All 4 Datasets

PHP programming language. Still, it is likely that we simply need to gather more samples for this dataset.

We do note that, using a Random Forest Classifier with ten-fold validation on the Digital Democracy dataset with PCA filtering applied, we achieved precision of .15, Recall of 0.15 , and an F0.5 score of .14.

\subsection{RQ3: How Does Our Model Compare To Others?}

\subsubsection{Comparison To Random Approach}

We trained a random classifier for each dataset, running each with one hundred times ten-fold validation. To construct the random classifiers, we iterated through each label in a dataset, computing eight random numbers to use as features and pairing them with each label. We then trained these random features using a J48 tree. The accuracy of the random classifier was identical to the proportion of $\mathrm{P}$ to $\mathrm{N}$ in the dataset. 
On the Accumulo and Isis datasets, the CCP classifier significantly outperformed a random classifier. For Accumulo, with an F0.1 score of .75 compared to a random F0.1 score of 0.02. Similarly on the Isis dataset, the classifier far outperformed random with an F0.1 score of .70 compared to 0.05. Because of this large edge over a random classifier, we can surmise that the Isis and Accumulo are predicting class-file changes in an intelligent manner. However, again, CCP failed to achieve better than random accuray on the DigitalDemocracy dataset, showing again that CCP probably needs a sizable amount of information in order to perform intelligent predictions.

\subsubsection{Comparison To Previous Studies}

To better judge the performance of the CCP classifier, we compared our classifier to previous impact analysis studies. This is not an apples to apples comparison, as we are unable to run CCP against the corpora of these studies, but it should shed some light on CCPs capabilities. Lindvalls precision, recall, and F0.1 score are by far the highest. This makes sense, since this was a study where domain expert humans performed impact analysis manually, reporting to researchers their predictions over time. CCP had the highest precision and F0.1 score of the non-manual systems, with a weighted average precision of .78, and an F0.1 score of .76. However, CCPs recall was far worse than all but the Ying study.

\subsubsection{Analysis}

CCPs precision was high compared to other studies, and extremely high in comparison to random. This shows that when CCP attempts to predict a class to change, it is very likely to predict the correct class. However, $\mathrm{CCP}$ was unable to match the recall of the Zimmerman paper. The research by Zimmerman et al. essentially uses class co-change history to create association rules. In contrast, we additionally leverage 


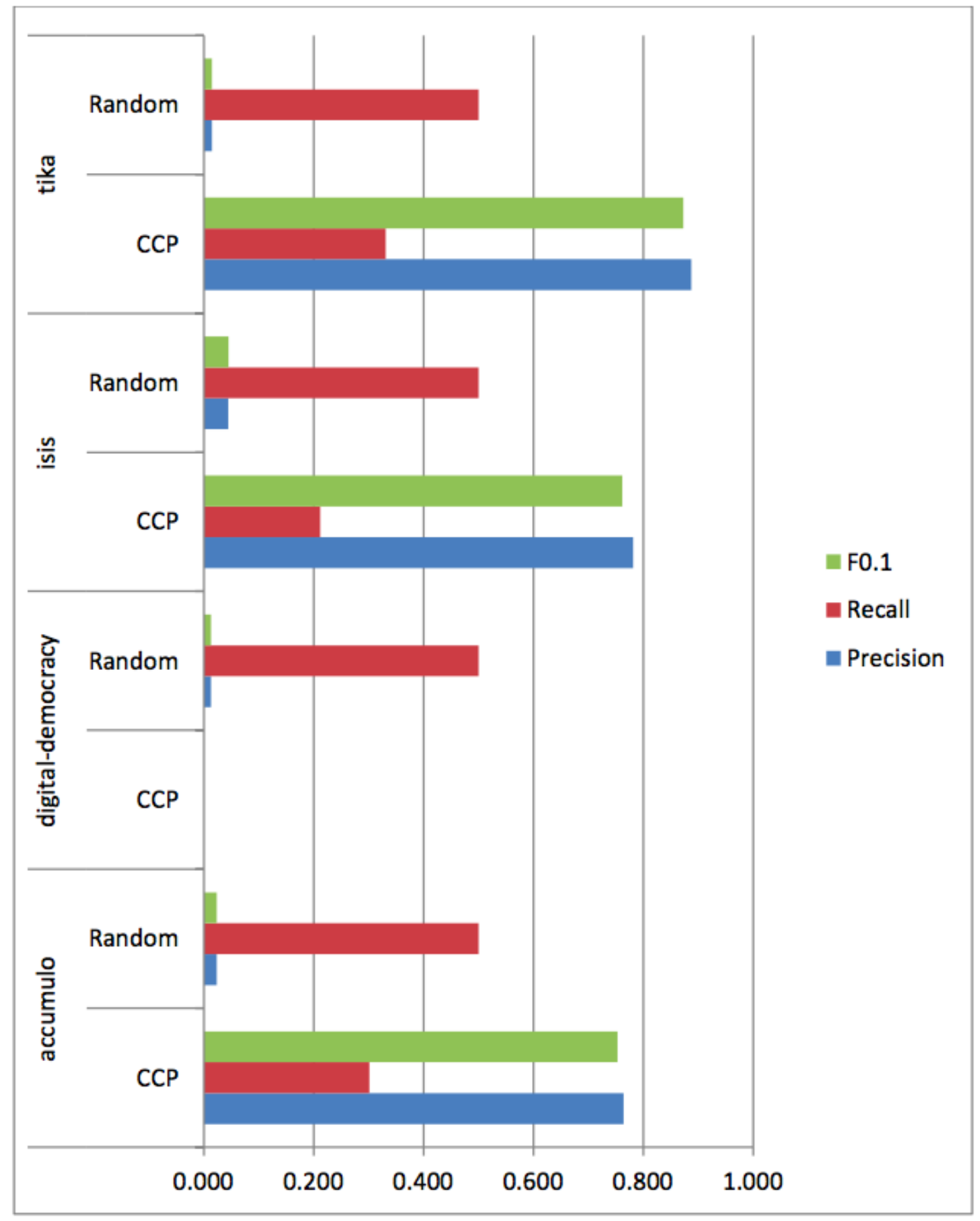

Figure 5.4: Comparison To Random 


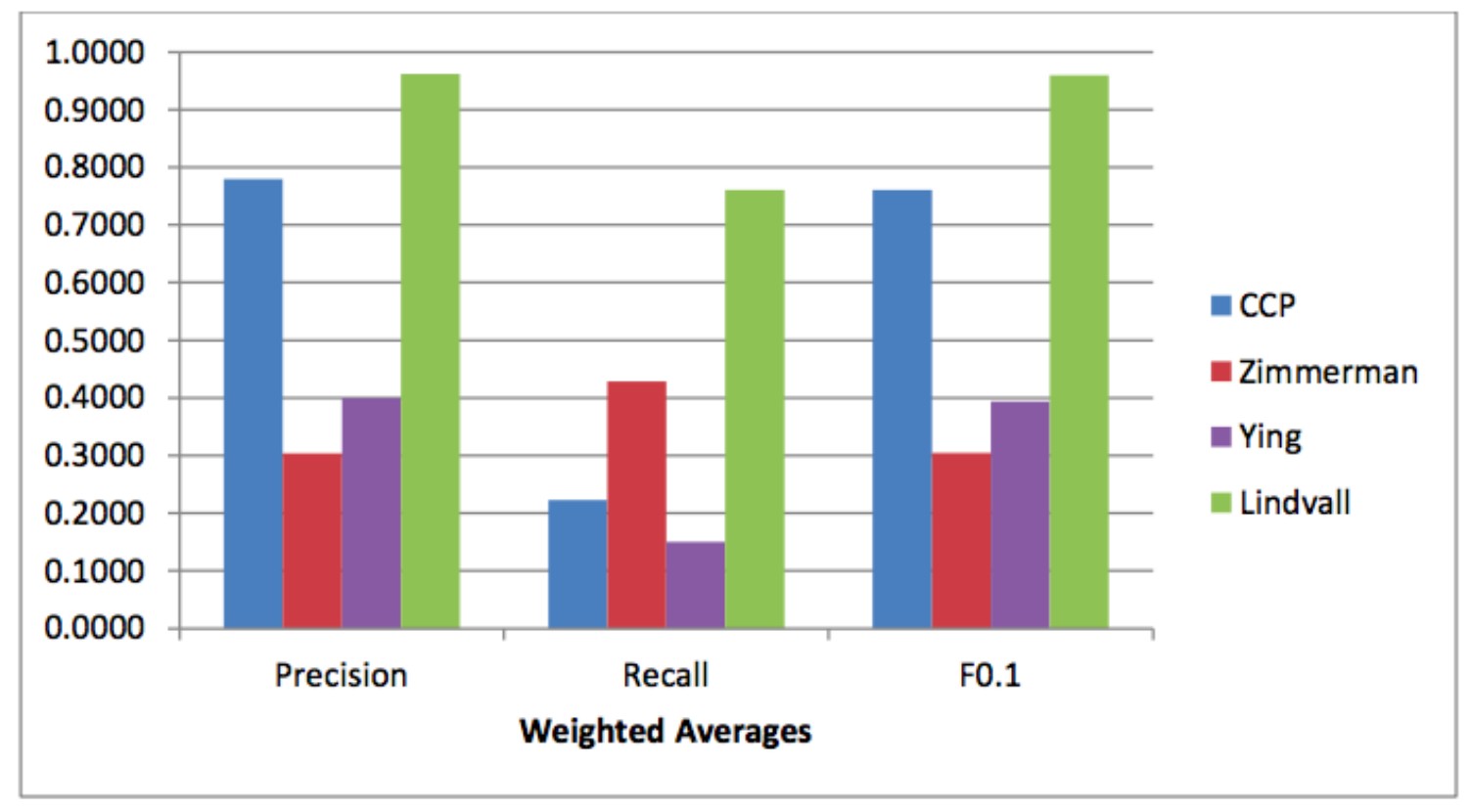

Figure 5.5: Comparison To Previous Studies

static analysis, requirement to requirement, and code to requirement similarity. The precision and recall of the Zimmerman et al. paper are thus impressive, and we will consider adding some co-change history functionality to future iterations of CCP. In order to improve recall, it is also likely that we need to perform some down-sampling of the data in order to increase the ratio of touched classes to non-touched classes in the datasets. 
Chapter 6

CONCLUSION AND FUTURE WORK

CCP provides a unique tool to mine SCM and project management repositories. CCP facilitates the building of machine learning models that, in our testing, have high precision overall.

CCP satisfies its main research questions and motivations in the following ways:

CCP Accomplished The Goal Of Developing A Configurable, Automated System To Datamine Project Management And SCM Repositories. CCP can be configured to use a variety of different metrics and analyze a variety of different software projects. Once configured properly, it can be run with limited human intervention.

CCP Has Accomplished The Goal Of Using A Unique Approach To Software Change Prediction. Though similar to some past impact analysis studies, CCP breaks new ground in that it combines elements of NLP, change history mining, static analysis, and object-oriented complexity analysis. We have provided original contributions in temporal locality and requirement to requirement comparison techniques.

RQ1: What Is The Most Accurate Configuration? We show that CCP has highest precision when utilizing a Bagging meta classifier with PCA applied to its features. This illustrates the usefulness of the features we have extracted. 
RQ2: What Is The Overall Accuracy Of Our Approach? CCP shows that it has high precision when run on multiple datasets, with a weighted F0.1 score of 0.8.

RQ3: How Does Our Model Compare To Others? We show that CCP has a high precision and F0.1 score in comparison to previous research.

\subsection{Threats To Validity}

It is important to note that this tool has only been tested on four different datasets, all of which are highly unbalanced, and only three of which only three produced an accurate classifier. It is likely that this tool needs to be run on the same datasets as similar impact analysis studies, such as the dataset used by Kim et al., to produce a conclusive comparison and show that we are not overfitting. However, CCP is quite tied to projects using Git and Jira, so running it against projects using other repositories would require significant development effort. As well, note that computing some of these metrics is expensive. Java projects require compiling all source code files in to byte code - which on larger projects can take a substantial amount of time. We found that many commits in all projects failed to build due to arcane configurations. SonarQube also failed to produce one or more metrics for many files across all of the different projects. Both of these factors reduced the number of samples available to us by at least half, which could skew our results.

\subsection{Future Work}

Our work on CCP is by no means complete. The following is a summary of plans for future development and analysis. 


\subsubsection{Deeper Analysis Of The Results}

We intend to perform further analysis on our existing data. It is likely that, with this analysis completed, we could improve the accuracy metrics of the CCP classifier. We could also determine what characteristics of the data are required for CCP to produce accurate results.

\subsubsection{Compare The Accuracy Of Estimates Made By Using A Single Set Of Metrics Versus The Complete Set}

When testing machine learning classifiers, it is a common practice to determine the set of features that are both the most independent, and produce the most accurate results [42]. We hypothesize that determining the most useful features will be extremely useful in supporting the future development of CCP, as we will be able to focus on improving existing sets of metrics that have all ready proved effective.

\subsubsection{Compare The Accuracy Of Estimates Made By Using Single Metrics Versus The Complete Set Of Metrics}

Likewise, researchers often try determining which individual features yield the most information gain in making classifications [42]. We could compare individual metrics both to each other and to the combined best set of metrics.

\subsubsection{Compare The Accuracy Of Our Approach When Using Only Data Related To The First Requirement Versus The Entire Dataset}

Since we are only gathering static and complexity metrics per release, rather than per requirement or per commit, it stands that these metrics will be more "stale" towards the end of a release's life cycle. It is thus apparent that the most "fresh" metrics will 
be available for the requirements created immediately after a new release. With this in mind, we hypothesize that training on requirements that are the very first after a release would produce more accurate results. The only caveat with this approach is that it results in a much smaller dataset to train on, so it is most suitable for software projects that are larger in terms of their number of classes, requirements, and commits.

\subsubsection{Find Better Temporal Locality Scores}

Since temporal locality was our most useful metric, it would be useful to try and produce more advanced temporal locality scores. Our temporal locality scores had a simple measure of time, in that we considered time as a measure of how many requirements had been submitted since the last requirement that touched a class. We could possibly improve upon this by using some unit of date-time measurement when computing the freshness of a touch, in a similar fashion to Bernstein et al. [20]. In fact, we could keep our existing measurements mostly in tact, but scale them by the number of weeks since a successful touch, instead of the number of requirements since a successful touch.

\subsubsection{Analyze The Locality Of Changes In The Different Datasets}

We realize that different datasets have relatively unique characteristics in terms of the frequency of commits and the frequency of closed requirements. These factors vary widely based on the style of the developers and management, and result in relatively unique temporal distances between completed requirements. While one dataset might have an average of at least a week between completed requirements, another might have an average of only a day. As such, we hypothesize that the temporal locality of 
a requirement should be weighted based on the average temporal distance between each requirement.

\subsubsection{Identify Other Dataset Characteristics Impacting The Accuracy Of Our Estimates}

We could likely find some additional dataset characteristics that are statistically relevant in producing accurate classification rates. We could determine additional characteristics such as the frequency with which new developers are added to a project

and the number of current branches of a project, then determine (using p-values and spearman scores) if those metrics corellate with improved accuracy measures.

\subsubsection{Approach Improvements}

\subsubsection{Leveraging Architecture Relations Information}

In 2005, Tsantalis et al. built a system to estimate how change-prone objects in a software project were [69]. They propose using four different axes to estimate how change-prone a class is.

- The inheritance axis, which tracks how many classes inherit from the class being analyzed. A change in the base class will likely require changes to all inheriting classes.

- The dependency axis, which measures dependencies of the class being analyzed on external classes and packages.

- The reference axis, which measures how often other classes instantiate or reference the class being analyzed. 
- The internal axis, which measures all possible causes of changes in a class, including method declarations and variables.

Tsantalis et al. compared the accuracy of using their metrics to that of CKJM, and reported much higher accuracy with their system [69]. We propose adding additional metrics to CCP's set of features based on Tsantalis's findings.

\subsubsection{Leveraging Co-changes Information}

Oftentimes when two classes are very highly coupled together, when a developer modifies one of the classes, she will have to modify the other class as well. Thus, when making a prediction for one class, it could be informative to look at all of the class co-changes that have occurred in the past. If coupled classes have been changed together in the past, it is likely that they will be changed together in the future, until such time as a developer has refactored the classes.

\subsubsection{Pre-process The Requirements For Their Quality}

There are a variety of qualities that typically indicate well-written requirements, such as being complete, unambiguous, correct, understandable, and consistent [32]. In recent years, researchers such as Gnova et al. have utilized NLP techniques and quality rules to produce automated tools that output requirements quality assessment

scores [40]. Kim et al. pre-processed requirements for their quality before performing a similar study to ours, and reported $70 \%$ accuracy. We hypothesize that well-written requirements are likely to be easier to link to a class change through NLP techniques. For example, in terms of $\mathrm{CCP}$, higher quality requirements should be more traceable, and thus have more consistent requirement to requirement and requirement to code similarity scores. 
We could leverage requirements quality in a few different ways. We could preprocess the requirements texts and produce a requirements reliability score. This could be another metric used to train a classifier, and a low reliability score could help the classifier avoid false positives. We could also simply filter out requirements with low requirements quality scores, and only train on high quality requirements.

\subsubsection{Leveraging Interconnected Frequency Information: Highest Frequency Of A Connected Class}

Though we included the afferent coupling and number of children metrics from CKJM, there is probably useful information to be gained by delving further into the dependencies between classes. A complex class that is in need of refactoring is likely highly coupled to at least one other class. We propose creating a new metric that takes the highest number of outgoing links to a particular class. This metric could possibly be leveraged and combined with the previously discussed co-change metric.

\subsubsection{Leveraging Interconnected Complexity Information}

In a similar fashion, we could traverse all connected classes for important metrics. For example, if a class is coupled to another class that is thousands of lines of code

long with extreme cyclomatic complexity, this could indicate that the class is prone to change, as it might require additional changes when the complex class is modified.

\subsubsection{Measuring All Metrics For Every Revision Rather Than For Every Release}

It is important to note that we gathered static metrics for every release, rather than for every commit. This is because collecting static metrics in java requires us to compile or build the project, which can take quite some time. Industrial projects 
often have hundreds of commits between releases, which means that the static metrics we gathered are often quite stale. It is thus easy to infer that static metrics that are more "fresh" will result in a more accurate classifier.

Since our approach only operated on one Git repository at a time, we could not parallelize the build tasks. In the future, the system could be modified to generate multiple copies of a git repository with multiple threads, and run builds using each of the different threads.

\subsubsection{Raised Research Questions: Measuring The Usefulness Of Our Approach In Practice}

Though we have tested the accuracy of our classifier using corpus cross-validation techniques, it would be important to perform a real-world usability study on CCP. This would likely require continued feedback from a development team in a similar fashion to that of Lindval et al's study on how accurately humans can predict software changes [57]. The development team could install CCP as a SonarQube plugin, then provide some additional survey data when opening each requirement.

\subsubsection{Release Planning: What is The Reduction Of Defects Provided By Minimizing The Number Of Developers Touching The Same Class?}

One of the tenets of agile programming is that smaller teams are generally more efficient than larger teams [14]. According to DAmbros et al., the number of developers touching a class impacts the defects injected [36]. The rationale is that over time in a large system, the same class can be touched by a wide range of requirements . New developers are brought in to work on different categories of requirements relating to the class, and those new developers will be more likely to inject defects into unfamiliar classes [36]. With this in mind, we could track the number of violations of classes in 
a project over time, as well as the number of developers that have recently modified that class. If there is statistically significant evidence that minimizing the number of developers touching a class leads to less defects, this could facilitate us adding a new metric to CCP: the number of developers that have recently modified a class.

\subsubsection{Refactoring: How Many Refactoring Decisions Could Be Avoided Or Improved By Using Our Approach?}

In addition to producing the classes that are predicted to be changed by a requirement, CCP could also display a brief change history for each class, as well as the number of defects. We hypothesize that developers can save effort by not refactoring a class that has not been changed often in the past, and will not be changed often in the future. We could then perform a usability study, and survey how often the CCP tool helps developers avoid costly refactoring.

\subsubsection{Effort Estimation: What Is The Improved Accuracy Of Effort Estima- tion Provided By Our Approach?}

Since the 1960's, there has been steady research involving the estimation of effort required to complete software tasks [33]. Using techniques such as the Constructive Cost Model (Cocomo) as well as regression, researchers attempt to predict the effort required to complete certain tasks based on a variety of variables, such as the developer's level of experience, the CPU constraints, and the size of the data or database to be leveraged [33]. Using the CCP tool, it would be informative to perform a study where the control group used a standard set of variables to predict the effort required to complete tasks, while the experimental group added metrics such as the complexity of the classes predicted to change by CCP. We could then determine how useful the CCP tool is in regards to improving effort estimation capabilities. 


\subsubsection{Defect Estimation: What Is The Improved Accuracy Of Defects Esti- mation Provided By Our Approach?}

When a developer reads a requirement, he or she will be prompted to answer a survey

question. The question will essentially ask what classes the developer thinks will be modified as a result of implementing the requirement. It has been shown that domainexpert level human developers often make errors in this regard [57]. We will compare the error rate of human predictions versus that of CCP.

\subsection{Final Thoughts}

CCP provides unique contributions to the field of impact analysis in software engineering, leveraging NLP, change history, and static analysis in one feature set. CCP's modular configuration allows future work on a variety of open-source repositories. In the future, we will likely run it on at least two more large projects. Though its recall is low, we believe this can be improved in future iterations.

We believe that this tool, released as a plugin to a system such as Jira or Git, could provide provide significant refactoring guidance to developers on large software projects. 


\section{BIBLIOGRAPHY}

[1] Apache accumulo project. http://isis.apache.org.

[2] Apache isis project. http://isis.apache.org.

[3] Apache maven project. http://maven.apache.org.

[4] Apache tika project. http://tika.apache.org.

[5] Cal Poly Github. http://www.github.com/CalPoly.

[6] Digital democracy. https://www.digital-democracy.org/.

[7] Findbugs. http://findbugs.sourceforge.net/.

[8] Git scm. http://www.git-scm.com.

[9] Jgit. https://eclipse.org/jgit/.

[10] Jira issue tracking software. http://www.jira.com.

[11] Semilar: A semantic similarity toolkit. http://www.semanticsimilarity.org/.

[12] Weka: Data mining software in java. http://www.cs.waikato.ac.nz/ ml/weka/.

[13] A. Abadi, M. Nisenson, and Y. Simionovici. A traceability technique for specifications. In The 16th IEEE International Conference on Program Comprehension, pages 103-112. IEEE, 2008.

[14] P. Abrahamsson, O. Salo, J. Ronkainen, and J. Warsta. Agile software development methods: Review and analysis, 2002.

[15] M. Acharya and B. Robinson. Practical change impact analysis based on static program slicing for industrial software systems. In Proceedings of the 33rd international conference on software engineering, pages 746-755. ACM, 2011. 
[16] M. N. Anyanwu and S. G. Shiva. Comparative analysis of serial decision tree classification algorithms. International Journal of Computer Science and Security, $3(3): 230-240,2009$.

[17] S. Arlot, A. Celisse, et al. A survey of cross-validation procedures for model selection. Statistics surveys, 4:40-79, 2010.

[18] A. Bagga and B. Baldwin. Entity-based cross-document coreferencing using the vector space model. In Proceedings of the 17th international conference on Computational linguistics-Volume 1, pages 79-85. Association for Computational Linguistics, 1998.

[19] A. L. Berger, V. J. D. Pietra, and S. A. D. Pietra. A maximum entropy approach to natural language processing. Computational linguistics, 22(1):39-71, 1996.

[20] A. Bernstein, J. Ekanayake, and M. Pinzger. Improving defect prediction using temporal features and non linear models. In Ninth international workshop on Principles of software evolution: in conjunction with the 6th ESEC/FSE joint meeting, pages 11-18. ACM, 2007.

[21] S. Bohner et al. Extending software change impact analysis into cots components. In Software Engineering Workshop, 2002. Proceedings. 27th Annual NASA Goddard/IEEE, pages 175-182. IEEE, 2002.

[22] S. A. Bohner. Software change impact analysis. 1996.

[23] L. Breiman. Bagging predictors. Machine learning, 24(2):123-140, 1996.

[24] L. Breiman. Random forests. Machine learning, 45(1):5-32, 2001.

[25] G. Campbell and P. P. Papapetrou. SonarQube in Action. Manning Publications Co., 2013. 
[26] G. Canfora and L. Cerulo. Impact analysis by mining software and change request repositories. In Software Metrics, 2005. 11th IEEE International Symposium, pages 9-pp. IEEE, 2005.

[27] S. R. Chidamber and C. F. Kemerer. A metrics suite for object oriented design. Software Engineering, IEEE Transactions on, 20(6):476-493, 1994.

[28] E. S. Cho, M. S. Kim, and S. D. Kim. Component metrics to measure component quality. In Software Engineering Conference, 2001. APSEC 2001. Eighth AsiaPacific, pages 419-426. IEEE, 2001.

[29] J. Cleland-Huang, A. Czauderna, M. Gibiec, and J. Emenecker. A machine learning approach for tracing regulatory codes to product specific requirements. In Proceedings of the 32nd ACM/IEEE International Conference on Software Engineering-Volume 1, pages 155-164. ACM, 2010.

[30] J. Cleland-Huang, O. C. Gotel, J. Huffman Hayes, P. Mäder, and A. Zisman. Software traceability: trends and future directions. In Proceedings of the on Future of Software Engineering, pages 55-69. ACM, 2014.

[31] C. Corley and R. Mihalcea. Measuring the semantic similarity of texts. In Proceedings of the ACL workshop on empirical modeling of semantic equivalence and entailment, pages 13-18. Association for Computational Linguistics, 2005.

[32] A. Davis, S. Overmyer, K. Jordan, J. Caruso, F. Dandashi, A. Dinh, G. Kincaid, G. Ledeboer, P. Reynolds, P. Sitaram, et al. Identifying and measuring quality in a software requirements specification. In Software Metrics Symposium, 1993. Proceedings., First International, pages 141-152. IEEE, 1993.

[33] K. Dejaeger, W. Verbeke, D. Martens, and B. Baesens. Data mining techniques for software effort estimation: a comparative study. Software Engineering, IEEE Transactions on, 38(2):375-397, 2012. 
[34] P. Domingos. A few useful things to know about machine learning. Communications of the ACM, 55(10):78-87, 2012.

[35] T. Dybå and T. Dingsøyr. Empirical studies of agile software development: A systematic review. Information and software technology, 50(9):833-859, 2008.

[36] M. DAmbros, M. Lanza, and R. Robbes. Evaluating defect prediction approaches: a benchmark and an extensive comparison. Empirical Software Engineering, 17(4-5):531-577, 2012.

[37] R. S. Engelmore and E. Feigenbaum. Expert systems and artificial intelligence. EXPERT SYSTEMS, 100:2, 1993.

[38] S. Eski and F. Buzluca. An empirical study on object-oriented metrics and software evolution in order to reduce testing costs by predicting change-prone classes. In Software Testing, Verification and Validation Workshops (ICSTW), 2011 IEEE Fourth International Conference on, pages 566-571. IEEE, 2011.

[39] D. Falessi, G. Cantone, and G. Canfora. Empirical principles and an industrial case study in retrieving equivalent requirements via natural language processing techniques. Software Engineering, IEEE Transactions on, 39(1):18-44, 2013.

[40] G. Génova, J. M. Fuentes, J. Llorens, O. Hurtado, and V. Moreno. A framework to measure and improve the quality of textual requirements. Requirements engineering, 18(1):25-41, 2013.

[41] C. Gupta and V. Gupta. Software change impact analysis: An approach to compute and prioritize impacted functions in software systems. International Journal of Systems and Service-Oriented Engineering (IJSSOE), 5(2):44-55, 2015.

[42] M. A. Hall. Correlation-based feature selection for machine learning. PhD thesis, The University of Waikato, 1999. 
[43] M. Hammad, M. L. Collard, J. Maletic, et al. Automatically identifying changes that impact code-to-design traceability. In Program Comprehension, 2009. ICPC'09. IEEE 17th International Conference on, pages 20-29. IEEE, 2009.

[44] M. A. Javed and U. Zdun. A systematic literature review of traceability approaches between software architecture and source code. In Proceedings of the 18th International Conference on Evaluation and Assessment in Software Engineering, page 16. ACM, 2014.

[45] J. J. Jiang and D. W. Conrath. Semantic similarity based on corpus statistics and lexical taxonomy. arXiv preprint cmp-lg/9709008, 1997.

[46] A. G. Jivani et al. A comparative study of stemming algorithms. Int. J. Comp. Tech. Appl, 2(6):1930-1938, 2011.

[47] I. Jolliffe. Principal component analysis. Wiley Online Library, 2002.

[48] M. Jureczko and L. Madeyski. A review of process metrics in defect prediction studies. Metody Informatyki Stosowanej, 5:133-145, 2011.

[49] E. Keenan, A. Czauderna, G. Leach, J. Cleland-Huang, Y. Shin, E. Moritz, M. Gethers, D. Poshyvanyk, J. Maletic, J. Huffman Hayes, et al. Tracelab: An experimental workbench for equipping researchers to innovate, synthesize, and comparatively evaluate traceability solutions. In Proceedings of the 34th International Conference on Software Engineering, pages 1375-1378. IEEE Press, 2012.

[50] D. Kim, Y. Tao, S. Kim, and A. Zeller. Where should we fix this bug? a twophase recommendation model. Software Engineering, IEEE Transactions on, 39(11):1597-1610, 2013. 
[51] S. Kim, T. Zimmermann, E. J. Whitehead Jr, and A. Zeller. Predicting faults from cached history. In Proceedings of the 29th international conference on Software Engineering, pages 489-498. IEEE Computer Society, 2007.

[52] S. N. Kim, O. Medelyan, M.-Y. Kan, and T. Baldwin. Semeval-2010 task 5: Automatic keyphrase extraction from scientific articles. In Proceedings of the 5th International Workshop on Semantic Evaluation, pages 21-26. Association for Computational Linguistics, 2010.

[53] B. A. Kitchenham, S. L. Pfleeger, L. M. Pickard, P. W. Jones, D. C. Hoaglin, K. El Emam, and J. Rosenberg. Preliminary guidelines for empirical research in software engineering. Software Engineering, IEEE Transactions on, 28(8):721$734,2002$.

[54] S. B. Kotsiantis, I. Zaharakis, and P. Pintelas. Supervised machine learning: A review of classification techniques, 2007.

[55] P. Kruchten, R. L. Nord, and I. Ozkaya. Technical debt: from metaphor to theory and practice. Ieee software, (6):18-21, 2012.

[56] S. Lehnert. A taxonomy for software change impact analysis. In Proceedings of the 12th International Workshop on Principles of Software Evolution and the 7th annual ERCIM Workshop on Software Evolution, pages 41-50. ACM, 2011.

[57] M. Lindvall and K. Sandahl. How well do experienced software developers predict software change? Journal of Systems and Software, 43(1):19-27, 1998.

[58] M. Lintean and V. Rus. Measuring semantic similarity in short texts through greedy pairing and word semantics. In Twenty-Fifth International FLAIRS Conference, 2012. 
[59] A. Mahmoud and N. Niu. Supporting requirements to code traceability through refactoring. Requirements Engineering, 19(3):309-329, 2014.

[60] A. McCallum, K. Nigam, et al. A comparison of event models for naive bayes text classification. In AAAI-98 workshop on learning for text categorization, volume 752, pages 41-48. Citeseer, 1998.

[61] M. Menéndez, J. Pardo, L. Pardo, and M. Pardo. The jensen-shannon divergence. Journal of the Franklin Institute, 334(2):307-318, 1997.

[62] M. Mirakhorli, Y. Shin, J. Cleland-Huang, and M. Cinar. A tactic-centric approach for automating traceability of quality concerns. In Proceedings of the 34th International Conference on Software Engineering, pages 639-649. IEEE Press, 2012.

[63] T. O'Brien et al. Developing with eclipse and maven.

[64] K. Papineni, S. Roukos, T. Ward, and W.-J. Zhu. Bleu: a method for automatic evaluation of machine translation. In Proceedings of the 40th annual meeting on association for computational linguistics, pages 311-318. Association for Computational Linguistics, 2002.

[65] C. Pettey. Gartner says worldwide software market grew 4.8 percent in 2013, March 2014. http://www.gartner.com/newsroom/id/2696317.

[66] V. Rus and M. Lintean. A comparison of greedy and optimal assessment of natural language student input using word-to-word similarity metrics. In Proceedings of the Seventh Workshop on Building Educational Applications Using NLP, pages 157-162. Association for Computational Linguistics, 2012.

[67] S. SonarSource. Sonarqube. Technical report, Technical report, last update: June, 2013. 
[68] D. Spinellis. ckjm chidamber and kemerer metrics software. Technical report, v 1.6. Technical report, Athens University of Economics and Business, 2005. http://-www. spinellis. gr/sw/ckjm, 2005.

[69] N. Tsantalis, A. Chatzigeorgiou, and G. Stephanides. Predicting the probability of change in object-oriented systems. Software Engineering, IEEE Transactions on, 31(7):601-614, 2005.

[70] S. Wold, K. Esbensen, and P. Geladi. Principal component analysis. Chemometrics and intelligent laboratory systems, 2(1-3):37-52, 1987.

[71] X. Wu, V. Kumar, J. R. Quinlan, J. Ghosh, Q. Yang, H. Motoda, G. J. McLachlan, A. Ng, B. Liu, S. Y. Philip, et al. Top 10 algorithms in data mining. Knowledge and information systems, 14(1):1-37, 2008.

[72] A. T. Ying, G. C. Murphy, R. Ng, and M. C. Chu-Carroll. Predicting source code changes by mining change history. Software Engineering, IEEE Transactions on, 30(9):574-586, 2004.

[73] J. Zheng, L. Williams, N. Nagappan, W. Snipes, J. P. Hudepohl, and M. A. Vouk. On the value of static analysis for fault detection in software. Software Engineering, IEEE Transactions on, 32(4):240-253, 2006. 
Appendix A

\section{EXAMPLE CLASS CHANGE}

To better understand the connection between a requirement and a commit, we will perform a case study of the modification history of the class PDF2XHTML.java in the Apache Tika project. With the first requirement, TIKA-158, we see that there is clear traceability between the PDF2XHTML.java class and the requirement text. PDF2XTHML.java contains numerous references to "PDF" and "PDFBox", so its code to requirement similarity is high. As well, it is coupled with PDFParser.java, as PDFParser.java creates a new PDF2XTHML object. Thus, we see them both change together.

The next requirement, TIKA-219, calls for a widespread modification and reorganization of dozens of classes. Because the requirement text is brief and imprecise, the traceability will be fairly low, and it may be difficult for our classifier to predict these changes.

On to TIKA-249. In this requirement, we see the requestor ask for modifications to virtually all of Tika's IO classes - one of which is PDF2XTHML.java. Almost all of the IO related classes reference files or throw IO exceptions, so the traceability is somewhat reasonable.

Figure A.1: First Modification

\begin{tabular}{|c|c|c|c|}
\hline \multirow[b]{2}{*}{ Reqld } & & & \\
\hline & Date & Other Changes & Text \\
\hline TIKA-158 & $\begin{array}{l}2009- \\
04-26 \\
\text { T11:3 } \\
5\end{array}$ & PDFParser.java & $\begin{array}{l}\text { The next PDFBox version will be an incubating } \\
\text { release from the Apache PDFBox podling. As } \\
\text { discussed in TIKA- } 114 \text { there are already some } \\
\text { fixes in PDFBox trunk that we could use, so we } \\
\text { should upgrade when the next release becomes } \\
\text { available. }\end{array}$ \\
\hline
\end{tabular}


Figure A.2: Large Amount Of Modifications

\begin{tabular}{l|l|l|l} 
TIKA-219 & 2009- & RTFParser.java,Tika & As discussed recently on the mailing list [1], it \\
& 04-28 & MimeKeys.java,Mim & would be good to split Tika to components based \\
T11:3 & $\begin{array}{l}\text { eTypestest.java, } \\
\text { index.apt,testPPT.pp }\end{array}$ & $\begin{array}{l}\text { on use cases and external dependencies. The } \\
\text { proposed split is ... }\end{array}$ \\
& $\begin{array}{l}\text { t,formats.apt,TypeD } \\
\text { etector.java,PDFPar } \\
\text { ser.java,OOXMLExtr } \\
\text { actor.java,ExcelExtr } \\
\text { actor.java }\end{array}$ &
\end{tabular}

Figure A.3: Many IO-related Modifications

TIKA-249

2009- ByteArrayOutputStr Commons IO is the only non-JDK dependency of

06-23 eam.java,Countingln

T21:3 putStream.java,Auto tika-core. To further simplify the dependency tree

5

DetectParser.java,

OpenOfficeParser.ja

and to help reduce the size of the standalone jar

va,CpioParser.java, I'd like to inline the key Commons IO classes we

OfflineContentHandl

er.java,ClosedInput

Stream.java,IOUtils.j

ava,HtmlParser.java

,IOExceptionWithCa

use.java,ImagePars

er.java,Bzip2Parser.j

ava,GzipParser.java

,CloseShieldlnputStr

eam.java,XMLParse

r.java,SecureConten

tHandlerTest.java,S

ecureContentHandle

r.java, TarParser.java 


\begin{tabular}{l|l|l|l} 
& \multicolumn{3}{|c}{ Figure A.4: Final Modification } \\
TIKA-292 & $\begin{array}{l}2009- \\
09-28\end{array}$ & $\begin{array}{l}\text { PDFBox 0.8 logs INFO messages for all PDF } \\
\text { T10:4 } \\
7\end{array} \mid$ & $\begin{array}{l}\text { primitives that are not enabled in the respective } \\
\text { PDFBox configuration. Many of these primitives } \\
\text { are explicitly not needed for text extraction, so } \\
\text { there's no point in logging so much about them. } \\
\text { Until this is fixed in PDFBox, we should work } \\
\text { around it in Tika. }\end{array}$ \\
\hline
\end{tabular}

With TIKA-292, we see another very specific request. The request again invokes the unique word-choice of PDFBox, which makes the request easily traceable to PDF2XHTML.java. We find that PDF2XTML.java is the only class that changes as a result of the requirement. 
Appendix B

\section{XML CONFIGURATION EXAMPLE}

This appendix contains an example of an XML configuration file.

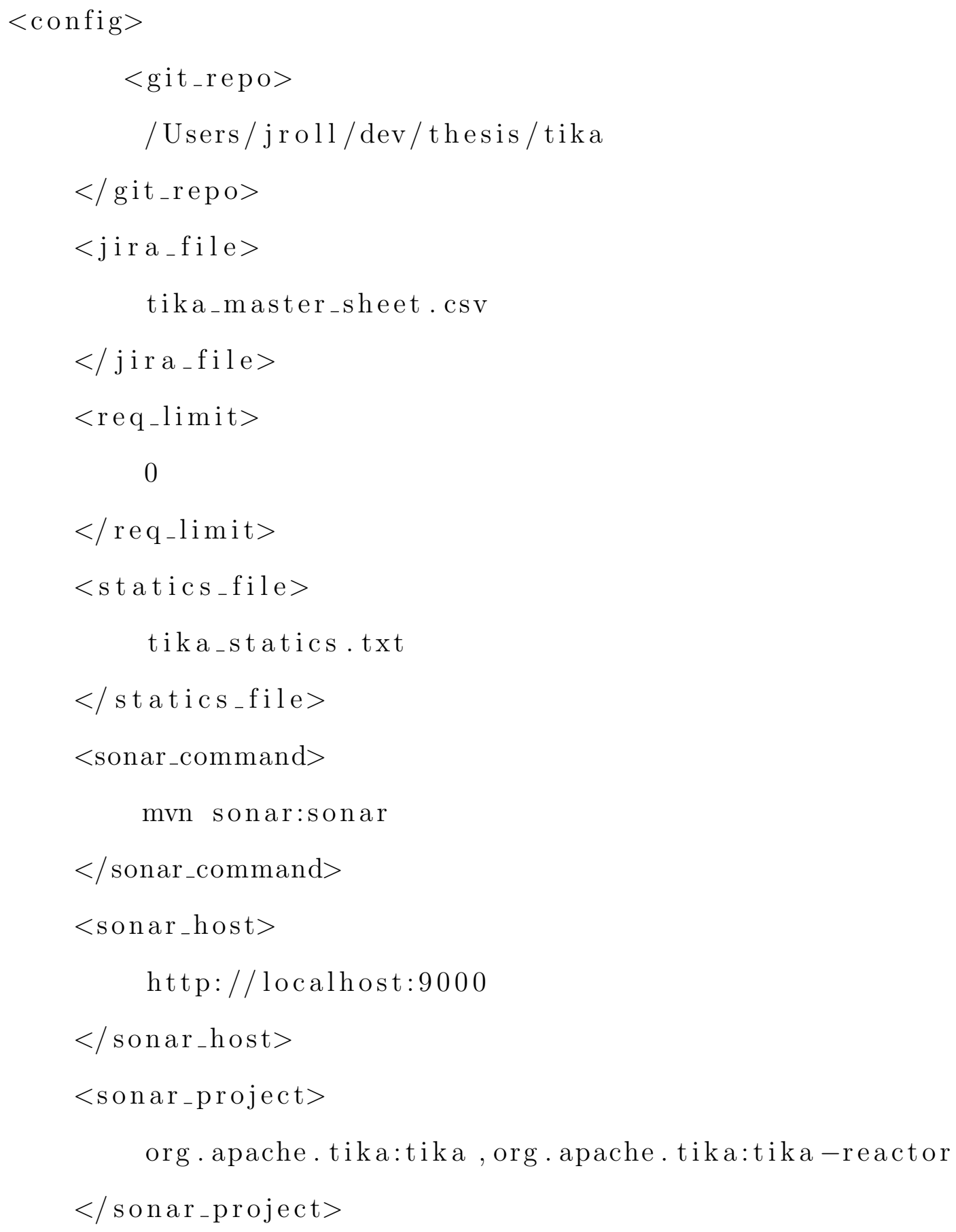




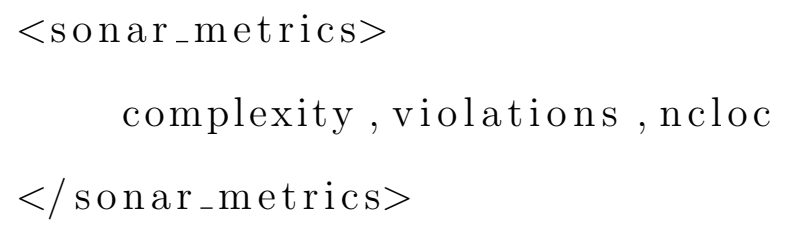




\section{Appendix C}

\section{WEKA CONFIGURATION}

This appendix includes weka configurations for each machine learning classifier. The

first column lists the formal name of the classifier, while the second column lists the specific flags and commands we used to execute the classifier inside weka. 
Table C.1: Weka Parameters

\begin{tabular}{|l|l|}
\hline Classifier & Parameters \\
\hline \hline Bagging & $\begin{array}{l}\text {-P } 100-\mathrm{S} 1-\mathrm{l} 10-\mathrm{W} \text { weka.classifiers.trees.REPTree } \\
-\mathrm{M} 2-\mathrm{V} 0.001-\mathrm{N} 3-\mathrm{S} 1-\mathrm{L}-1\end{array}$ \\
\hline Bayes Net & $\begin{array}{l}\text {-D -Q weka.classifiers.bayes.net. search.local.K2 -P } \\
\text {-S BAYES -E weka.classifiers.net.estimate. } \\
\text { SimpleEstimator-A 0.5 }\end{array}$ \\
\hline J48 & $-\mathrm{C} 0.25$-M 2 \\
\hline Logistic & $-\mathrm{R} 1.0 \mathrm{E}-8$-M -1 \\
\hline Naive Bayes & Default configuration- \\
\hline Random Forest & -1 100 -K 0 -S 1 \\
\hline
\end{tabular}




\section{Appendix D}

\section{FEATURES}

This appendix includes a list of each feature used in training the machine learning classifier. Each feature group is specified in a separate table, which includes the name of the feature, as well as a brief description of the feature's technical details. 
Table D.1: Requirements To Code Metrics

\begin{tabular}{|l|l|}
\hline Method Name & Brief Description \& Tools/Refs \\
\hline Vector Space Model & $\begin{array}{l}\text { Compares source text T1 and target code C1 as vectors } \\
\text { in the space constructed by index terms. Frequency of } \\
\text { terms occurrence in the texts and in the collection of } \\
\text { documents are normally used for weighting the terms. }\end{array}$ \\
\hline Jensen Shannon & $\begin{array}{l}\text { Compare T1 and C1 as two probability distributions of } \\
\text { terms. It is based on the Kullback-Leibler divergence } \\
\text { which measures the average inefficiency in using one } \\
\text { distribution to code for another. [61] }\end{array}$ \\
\hline
\end{tabular}


Table D.2: Textual Similarity Methods To Compute Requirements To Class Associations

\begin{tabular}{|c|c|}
\hline Method Name & Brief Description \& Tools/Refs \\
\hline $\begin{array}{l}\text { VSM: } \\
\text { Vector Space Model }\end{array}$ & $\begin{array}{l}\text { Compares source text } \mathrm{T} 1 \text { and target text } \mathrm{T} 2 \text { as } \\
\text { vectors in the space constructed by index terms. } \\
\text { Frequency of terms occurrence in the texts and in the } \\
\text { collection of documents are normally used for } \\
\text { weighting the terms[18]. }\end{array}$ \\
\hline $\begin{array}{l}\text { JSD: } \\
\text { Jensen Shannon } \\
\text { Divergence }\end{array}$ & $\begin{array}{l}\text { Compare } \mathrm{T} 1 \text { and } \mathrm{T} 2 \text { as two probability distributions } \\
\text { of terms. It is based on the Kullback-Leibler } \\
\text { divergence which measures the average inefficiency in } \\
\text { using one distribution to code for another. [61] }\end{array}$ \\
\hline $\begin{array}{l}\text { GC: } \\
\text { Greedy Comparator }\end{array}$ & $\begin{array}{l}\text { Term-to-term semantic similarity measures are } \\
\text { computed first using WordNet. Each term in T1 is } \\
\text { then paired with every term in T2 and the maximum } \\
\text { similarity score is greedily retained. A weighted sum } \\
\text { is then calculated as the text similarity. [66] }\end{array}$ \\
\hline $\begin{array}{l}\text { OPC: } \\
\text { Optimum Comparator }\end{array}$ & $\begin{array}{l}\text { Term-to-term semantic similarity measures are } \\
\text { computed first as in Greedy Comparator. Each term } \\
\text { in } \mathrm{T} 1 \text { is then paired with the term in } \mathrm{T} 2 \text { so that } \\
\text { global maximum similarity score is achieved. [66] }\end{array}$ \\
\hline $\begin{array}{l}\text { CMC: Corley Mihalcea } \\
\text { Comparator }\end{array}$ & $\begin{array}{l}\text { Terms in } \mathrm{T} 1 \text { are compared with terms in } \mathrm{T} 2 \text { with the } \\
\text { same Part-of-speech (POS) tags. For nouns and } \\
\text { verbs, the semantic similarity is used while lexical } \\
\text { similarity is used for all other POS groups. Inverse } \\
\text { document frequency is used for weighting terms when } \\
\text { calculate their sum. [66] }\end{array}$ \\
\hline
\end{tabular}


Table D.3: Temporal Locality Metrics

\begin{tabular}{|l|l|}
\hline Method Name & Brief Description \& Tools/Refs \\
\hline Simple & $\begin{array}{l}\text { Number of classes touched divided by touch } \\
\text { opportunities for the class. }\end{array}$ \\
\hline Linear & $\begin{array}{l}\text { Number of classes touched, weighted by their recency, } \\
\text { divided by the total touch opportunities. }\end{array}$ \\
\hline Logarithmic & $\begin{array}{l}\text { Number of classes touched, weighted logarithmically by } \\
\text { their recency, divided by total touch opportunities }\end{array}$ \\
\hline
\end{tabular}


Table D.4: Source Code Coupling, Cohesion, And Complexity Metrics

\begin{tabular}{|c|c|c|}
\hline Metric & Tool & Description \\
\hline Code Violations & Sonar & $\begin{array}{l}\text { Defines rules for detecting code smells. The metric counts } \\
\text { the number of code smell violations [67]. (Error Propensity) }\end{array}$ \\
\hline KeyWord Count & Sonar & $\begin{array}{l}\text { Counts number of key-words (e.g. if, else, for, while, etc. } \\
\text { [67] (Size, Complexity) }\end{array}$ \\
\hline $\begin{array}{l}\text { LOC: } \\
\text { Lines of Code }\end{array}$ & CKJM & $\begin{array}{l}\text { Counts number of lines of code (excluding white space and } \\
\text { brackets). (Size) }\end{array}$ \\
\hline $\begin{array}{l}\text { WMC: Weighted } \\
\text { Methods per Class }\end{array}$ & CKJM & $\begin{array}{l}\text { Sums number of methods per class, weighted by the } \\
\text { cyclomatic complexity for each method [28]. (Size, } \\
\text { Complexity) }\end{array}$ \\
\hline $\begin{array}{l}\text { DIT: } \\
\text { Depth of Inheritance } \\
\text { Tree }\end{array}$ & CKJM & $\begin{array}{l}\text { Measures the number of levels in a class's inheritance tree. } \\
\text { DIT is an indicator of code-reuse [28]. (Coupling) }\end{array}$ \\
\hline $\begin{array}{l}\text { NOC: } \\
\text { Number of Children }\end{array}$ & CKJM & $\begin{array}{l}\text { Measures the number of times a class has been subclassed } \\
{[28] . \text { (Coupling) }}\end{array}$ \\
\hline $\begin{array}{l}\text { CBO: Coupling } \\
\text { between Object Classes }\end{array}$ & CKJM & $\begin{array}{l}\text { Measures the number of non-inheritance based associations } \\
\text { to other classes [28]. (Coupling) }\end{array}$ \\
\hline $\begin{array}{l}\text { RFC: } \\
\text { Response for a Class }\end{array}$ & CKJM & $\begin{array}{l}\text { Counts the methods that may be invoked by a class in } \\
\text { response to an event [28]. (Coupling) }\end{array}$ \\
\hline $\begin{array}{l}\text { LCOM: } \\
\text { Lack of Cohesion of } \\
\text { Metrics }\end{array}$ & CKJM & $\begin{array}{l}\text { Measures the extent to which methods in a class are } \\
\text { internally cohesive [28]. (Cohesion) }\end{array}$ \\
\hline $\begin{array}{l}\text { Ca: } \\
\text { Afferent Couplings }\end{array}$ & CKJM & $\begin{array}{l}\text { Measures the number of direct references from other classes } \\
{[28] . \text { (Coupling) }}\end{array}$ \\
\hline $\begin{array}{l}\text { NPM: Number of } \\
\text { Public Methods }\end{array}$ & CKJM & $\begin{array}{l}\text { Measures the number of publicly exposed methods [28]. } \\
\text { (Cohesion, Coupling) }\end{array}$ \\
\hline
\end{tabular}

